\title{
Identification of Two Functionally Distinct Endosomal Recycling Pathways for Dopamine $\mathrm{D}_{2}$ Receptor
}

\author{
Yun Li, ${ }^{1}$ Brittany D. Roy, ${ }^{1}$ Wei Wang, ${ }^{1}$ Li Zhang, ${ }^{2}$ Lifeng Zhang, ${ }^{1}$ Stephen B. Sampson, ${ }^{1}$ Yupeng Yang, ${ }^{2}$ and Da-Ting Lin ${ }^{1}$ \\ ${ }^{1}$ The Jackson Laboratory, Bar Harbor, Maine 04609 and ${ }^{2}$ CAS Key Laboratory of Brain Function and Diseases, School of Life Science, University of Science \\ and Technology of China, Hefei, Anhui 230027, China
}

\begin{abstract}
Dopamine $\mathrm{D}_{2}$ receptor (DRD2) is important for normal function of the brain reward circuit. Lower DRD2 function in the brain increases the risk for substance abuse, obesity, attention deficit/hyperactivity disorder, and depression. Moreover, DRD2 is the target of most antipsychotics currently in use. It is well known that dopamine-induced DRD2 endocytosis is important for its desensitization. However, it remains controversial whether DRD2 is recycled back to the plasma membrane or targeted for degradation following dopamine stimulation. Here, we used total internal reflection fluorescent microscopy (TIRFM) to image DRD2 with a superecliptic pHluorin tagged to its $\mathrm{N}$ terminus. With these technical advances, we were able to directly visualize vesicular insertion events of DRD2 in cultured mouse striatal medium spiny neurons. We showed that insertion of DRD2 occurs on neuronal somatic and dendritic surfaces. Lateral diffusion of DRD2 was observed following its insertion. Most importantly, using our new approach, we uncovered two functionally distinct recycling pathways for DRD2: a constitutive recycling pathway and a dopamine activity-dependent recycling pathway. We further demonstrated that Rab4 plays an important role in constitutive DRD2 recycling, while Rab11 is required for dopamine activitydependent DRD2 recycling. Finally, we demonstrated that the two DRD2 recycling pathways play distinct roles in determining DRD2 function: the Rab4-sensitive constitutive DRD2 recycling pathway determines steady-state surface expression levels of DRD2, whereas the Rab11-sensitive dopamine activity-dependent DRD2 recycling pathway is important for functional resensitization of DRD2. Our findings underscore the significance of endosomal recycling in regulation of DRD2 function.
\end{abstract}

\section{Introduction}

The mesolimbic dopamine system is a central component of the brain reward circuit (Wise, 2008). Substantial evidence suggests that lower dopamine (DA) $\mathrm{D}_{2}$ receptor (DRD2) function in the striatum increases risk for substance abuse (Volkow et al., 2009), obesity (Wang et al., 2002; Kenny, 2011), attention deficit/hyperactivity disorder (Blum et al., 1996), and depression (Park et al., 2005; Lawford et al., 2006). Moreover, abnormal elevated function of DRD2 is thought to contribute to the positive symptoms of schizophrenia (Carlsson, 1988; Karam et al., 2010). For this reason, most antipsychotics currently in use are DRD2 antagonists. The function of DRD2 is determined by its levels on the plasma membrane (PM) (Lin et al., 2001, 2002; Binda et al., 2002; Free et al., 2007; Kim et al., 2008a,b; Tirotta et al., 2008; Genedani

\section{Received Dec. 30, 2011; revised April 8, 2012; accepted April 10, 2012.}

Author contributions: Y.L. and D.-T.L. designed research; Y.L. performed research; Y.Y. contributed unpublished reagents/analytic tools; B.D.R., W.W., Li Zhang, and Lifeng Zhang analyzed data; Y.L., S.B.S., and D.-T.L. wrote the paper.

This work was supported by startup funds from The Jackson Laboratory. We thank Dr. Richard L. Huganir (Department of Neuroscience, Johns Hopkins University School of Medicine/ HHMI) for insightful discussion during the initial phase of the project and for providing polyclonal and monoclonal GFP antibodies, and Rab4DN and Rab11DN DNA. We thank Jeff Forthofer and Dave Davis of the Jackson Laboratory Infrastructure Services for their help in the initial setting up of our custom-built TIRF microscope. We thank Dr. Zhong-wei Zhang for providing the DRD2-Cre mouse strain, and Drs. Zhong-wei Zhang and Robert Burgess for critical reading of this manuscript.

The authors declare no competing financial interests.

Correspondence should be addressed to Dr. Yun Li or Dr. Da-Ting Lin, The Jackson Laboratory, 600 Main Street, ERB Box 969, Bar Harbor, ME 04609. E-mail: yun@jax.org or dlin@jax.org.

DOI:10.1523/JNEUROSCI.0008-12.2012

Copyright $\odot 2012$ the authors $\quad 0270-6474 / 12 / 327178-13 \$ 15.00 / 0$ et al., 2010), and DRD2 endocytosis plays an important role in its desensitization following DA stimulation (Kim et al., 2001; Jeanneteau et al., 2004; Kabbani et al., 2004; Macey et al., 2004; Namkung and Sibley, 2004; Sugiura et al., 2004; Bartlett et al., 2005; Genedani et al., 2005; Torvinen et al., 2005; Paspalas et al., 2006; Iizuka et al., 2007; Kim, 2008; Xiao et al., 2009; Celver et al., 2010; Shimokawa et al., 2010). However, the fate of DRD2 following DA-induced endocytosis remains controversial. DRD2 was suggested to target primarily to degradation pathways following DA-induced endocytosis (Bartlett et al., 2005). Recently, dysbindin has been implicated in targeting DRD2 to degradation pathways (Ji et al., 2009; Marley and von Zastrow, 2010). However, evidence also exists that supports DRD2 recycling following its endocytosis (Vickery and von Zastrow, 1999; Namkung et al., 2009). If endosomal recycling of DRD2 does take place, it is likely to be important in determining the abundance of DRD2 on the PM. Most importantly, targeting endosomal recycling of DRD2 to manipulate the number of DRD2 receptors on the PM offers a potentially effective therapeutic strategy for psychiatric disorders with DRD2 dysfunction. Determining whether endosomal recycling of DRD2 exists, and, if it does exist, elucidating how endosomal recycling of DRD2 is regulated, represents an important step toward this ultimate therapeutic goal. The highest DRD2 expression is detected in the striatum, and $>90 \%$ of neurons in the striatum are medium spiny neurons (MSNs) (Bunzow et al., 1988; Missale et al., 1998). Hence, primary cultured MSNs represent an excellent cellular model system for elucidating the molecular and cellular mechanisms governing DRD2 trafficking and 
function. Here, we directly visualized DRD2 insertion to the PM of MSNs by using a new strategy to label DRD2 and by using total internal reflection fluorescent microscopy (TIRFM). Using our new approach, we uncovered two functionally distinct recycling pathways for DRD2: a Rab4-sensitive constitutive recycling pathway that determines steady-state surface expression of DRD2 and a Rab11-sensitive DA activity-dependent recycling pathway that is important for functional resensitization of DRD2. Our findings underscore the significance of endosomal recycling in regulation of DRD2 function.

\section{Materials and Methods}

All restriction enzymes were obtained from New England BioLabs. Chemicals were obtained from Thermo Fisher Scientific. Tissue culture supplies are from Invitrogen. HEK 293 cells were transfected using Lipofectamine 2000 (Invitrogen) per the manufacturer's recommendation. The long isoform of human DRD2 (DRD2L) cDNA was obtained from Open Biosystems. The short isoform of human DRD2 (DRD2S) was generated by PCR. DNA sequencing was performed in The Jackson Laboratory Scientific Services' DNA sequencing facility.

$p H-D R D 2$ construction. To generate $\mathrm{N}$-terminal superecliptic pHluorin-tagged DRD2, we first subcloned superecliptic pHluorin into the pRK5 vector. No cleavable signal peptide was identified on DRD2; therefore, to ensure correct targeting of pHluorin and the $\mathrm{N}$ terminus of DRD2 to the endoplasmic reticulum lumen following protein translation, a cleavable signal peptide sequence, MPYIFAFFCTGFLGAVVGAN, was added to the $\mathrm{N}$ terminus of superecliptic pHluorin. A 10-glycine linker was inserted between the signal peptide and the $\mathrm{N}$ terminus of pHluorin. Another 10-glycine linker was inserted between the $\mathrm{C}$ terminus of pHluorin and the $\mathrm{N}$ terminus of DRD2. DRD2L or DRD2S was cloned in frame into the $\mathrm{pRK} 5$ vector fused to pHluorin behind the glycine linker. The final cDNA constructs (pRK5$\mathrm{pH}-\mathrm{DRD} 2 \mathrm{~L}$ and $\mathrm{pRK} 5-\mathrm{pH}-\mathrm{DRD} 2 \mathrm{~S}$ ) were sequenced to ensure that no mutation was introduced by PCR.

To generate the pCAG-LoxP-Neomycin-LoxP-pH-DRD2L (pCALNL-pHDRD2L) construct, we obtained pCALNL-DsRed from AddGene and replaced DsRed on this vector with $\mathrm{pH}$-DRD2L. The final construct contains a CAG promoter, followed by a floxed neomycin cassette. The $\mathrm{pH}-\mathrm{DRD} 2 \mathrm{cDNA}$ was placed behind the floxed neomycin cassette. In the absence of Cre recombinase activity, neomycin is translated, thereby preventing translation of $\mathrm{pH}-\mathrm{DRD} 2 \mathrm{~L}$ when transfected in wild-type neurons (data not shown). In the presence of Cre, the neomycin cassette is removed, allowing translation and expression of $\mathrm{pH}-\mathrm{DRD} 2 \mathrm{~L}$. Expressing this construct in neurons cultured from DRD2-Cre embryos will result in specific expression of $\mathrm{pH}-\mathrm{DRD} 2 \mathrm{~L}$ in $\mathrm{D}_{2}$-type MSNs.

DRD2 Cre mice. The DRD2 Cre mouse strain STOCK $\mathrm{Tg}(\mathrm{Drd} 2-$ cre)ER44Gsat/Mmucd was obtained from Mutant Mouse Regional Resource Centers and was backcrossed to the C57BL/6J genetic background for more than five generations. To perform the experiments shown in Fig. $1 \mathrm{~K}$, we first crossed heterozygous DRD2 Cre mice and used real-time PCR to identify homozygous DRD2 Cre mice. We then crossed homozygous male DRD2 Cre mice with C57BL/6J females, a strategy that ensures that all $\mathrm{D}_{2}$-type MSNs in the resulting embryos are labeled with Cre. We used these embryos for our neuronal culture and transfected with pCALNL-pH-DRD2L or pRK5-pH-DRD2L. When we crossed homozygous male DRD2 Cre mice with homozygous female B6;129S6-

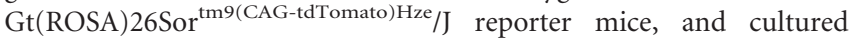
neurons from these embryos, only a portion of MSNs were labeled with tdTomato (data not shown), suggesting that MSNs in culture can also be categorized as $\mathrm{D}_{1^{-}}$or $\mathrm{D}_{2}$-type MSNs. Due to the interference of tdTomato fluorescence in our $\mathrm{pH}$-DRD2 TIRF imaging experiments, pCALNL-pH-DRD2L or regular pRK5-pH-DRD2L constructs were used to transfect DRD2-Cre MSNs for comparison of pH-DRD2L insertion in $\mathrm{D}_{2}$-type MSNs versus mixed MSNs.

cAMP accumulation assay. The cAMP accumulation assay for determining DRD2 function was performed using the LANCE Ultra cAMP Detection Kit from PerkinElmer using the protocols provided by the manufacturer. In brief, a cAMP standard curve was generated using reagents provided in the detection kit. The final cAMP concentration (in $\mathrm{mol} / \mathrm{L})$ was $1 \times 10^{-6}, 3 \times 10^{-7}, 1 \times 10^{-7}, 3 \times 10^{-8}, 1 \times 10^{-8}, 3 \times$ $10^{-9}, 1 \times 10^{-9}, 3 \times 10^{-10}, 1 \times 10^{-10}, 3 \times 10^{-1}, 1 \times 10^{-11}, 0$. The standard curve was fitted to an equation: $\log$ (agonist) versus response minus variable slope in Prism (GraphPad Software). The equation is as follows: $Y=$ bottom $+($ top - bottom $) /\left(1+10 \wedge\left[\left(\operatorname{LogEC}_{50}-X\right){ }^{*}\right.\right.$ Hill slope $\left.]\right)$. In this equation, $Y$ represents time-resolved fluorescence resonance energy transfer (TR-FRET) signals measured at $665 \mathrm{~nm}$, and $\mathrm{X}$ represents $\log (\mathrm{cAMP})$. Fitting the cAMP standard curve with the above equation gave the numerical values of bottom, top, $\operatorname{LogEC}_{50}$, and Hill slope, and yielded the following equation: $Y=1234+57751 /\left(1+10^{(-8.796-\mathrm{X}) *(-1.749)}\right)$. By applying these numerical values to the above equation, we calculated the true cAMP concentration from the TR-FRET signals measured at $665 \mathrm{~nm}$.

To measure DRD2 activity, HEK 293 cells were transfected with vector alone, DRD2, or pH-DRD2. Since activation of DRD2 results in inhibition of cAMP accumulation, we stimulated HEK 293 cells with $3 \mu \mathrm{M}$ of forskolin for $30 \mathrm{~min}$ to increase the cAMP concentration. We simultaneously treated HEK 293 cells with three physiological concentrations of dopamine $(1,10$, and $100 \mathrm{~nm})$ for $30 \mathrm{~min}$. Following dopamine stimulation, cAMP accumulation was assayed using the reagents provided in the detection kit and using a VICTOR X3 Multilabel Plate Reader (PerkinElmer). The TR-FRET signals obtained from the plate reader were then converted into cAMP concentrations using the equation described above. The cAMP concentrations were further normalized to samples not treated with dopamine for each group.

Neuronal culture. Striatum from embryonic day 16 (E16) mice were dissected out under a dissection microscope in cold artificial CSF (ACSF) containing the following (in $\mathrm{mm}$ ): $119 \mathrm{NaCl}, 5 \mathrm{KCl}, 1 \mathrm{MgCl}_{2}, 30$ dextrose, 25 HEPES, pH 7.4, without calcium. Striatal neurons were dissociated with papain in ACSF at $37^{\circ} \mathrm{C}$ for $15 \mathrm{~min}$ and seeded on $25 \mathrm{~mm}$ coverslips (size \#1.5) precoated with poly-L-lysine ( $0.1 \mathrm{M}$ in borate buffer, $\mathrm{pH} 8.0$ ). The plating medium was Neurobasal medium containing $50 \mathrm{U} / \mathrm{ml}$ penicillin, $50 \mu \mathrm{g} / \mathrm{ml}$ streptomycin, and $2 \mathrm{~mm}$ GlutaMAX, supplemented with $2 \%$ B-27 and 5\% heat-inactivated horse serum (Invitrogen). Twentyfour hours after initial plating, the original plating medium was replaced with fresh plating medium, and neurons were supplied with a feeder layer of glia growing on a PICM 03050 culture insert (Millipore). Neurons were then fed twice weekly with Neurobasal media (see above) supplemented with $2 \%$ B-27. Neurons were used for imaging experiments between 8 and 12 days in vitro.

Neuronal transfection. Lipofectamine 2000 (Invitrogen) was used for neuronal transfection. For each $25 \mathrm{~mm}$ coverslip, $2 \mu \mathrm{l}$ of Lipofectamine 2000 was used. One microgram $(1 \mu \mathrm{g})$ of $\mathrm{pH}-\mathrm{DRD} 2 \mathrm{cDNA}$ was used in all experiments. For experiments coexpressing $\mathrm{pH}-\mathrm{DRD} 2$ with dominant-negative Rab4 (Rab4DN) or dominant-negative Rab11 (Rab11DN), an additional $1 \mu \mathrm{g}$ of empty vector, or of a cDNA vector expressing Rab4DN or Rab11DN, was cotransfected with pH-DRD2. Lipofectamine 2000 and DNA were first each diluted with $200 \mu \mathrm{l}$ of Neurobasal medium without any supplement and were then mixed. The mixture was then let to stand for $20 \mathrm{~min}$ before being applied to neurons. Before application of the Lipofectamine/DNA complex to neurons, half of the culture medium was removed and mixed with an equal volume of new Neurobasal medium supplemented with B-27. This medium was saved for use with neurons after transfection. The Lipofectamine/ DNA mixture was then added to neurons and incubated for 2-4 h. Following incubation, the transfection medium containing the Lipofectamine/DNA complex was replaced with the medium prepared before transfection. Neurons were used for experiments $24-72 \mathrm{~h}$ following transfection.

TIRFM imaging. The TIRFM system is constructed based on a manual Zeiss AxioObserver microscope (Carl Zeiss MicroImaging). The excitation laser is a Newport $488 \mathrm{~nm} / 100 \mathrm{~mW}$ Cyan Laser System. The laser is coupled to a Zeiss TIRF slider via a KineFLEX-P-2-S-488-640-0.7FCP-P2 optical fiber (Point Source). A Z488RDC dichroic mirror (Chroma Technology) was used to reflect the incoming laser onto a Zeiss $\alpha$-Plan $100 \times$ objective lens [numerical aperture (NA), 1.46]. An ET525/50 emission filter was used for GFP fluorescence detection (Chroma Technology). An Evolve Electron Multiplying Charge Coupled Device (EMCCD) camera (Photometrics) was used as the detector. A $2.5 \times$ relay lens was positioned between the microscope camera port and 
the camera to achieve optimal spatial resolution $(0.064 \mu \mathrm{m}$ per pixel when using the $100 \times, 1.46$ NA objective). The camera was maintained at $-80^{\circ} \mathrm{C}$ during imaging experiments. A Uniblitz LS6 shutter controlled by a VMM-D3 controller (Vincent Associates) was integrated between the laser head and fiber launcher. Data were acquired using $\mu$-Manager software (Edelstein et al., 2010) (http://www.micro-manager.org/). All imaging experiments were performed at $37^{\circ} \mathrm{C}$ in ACSF solution containing $2 \mathrm{mM} \mathrm{CaCl}_{2}$. Camera exposure was set at $100 \mathrm{~ms}$, and the acquisition rate was 10 images per second $(10 \mathrm{~Hz})$. EMCCD gain was set at the maximum. Recordings were analyzed using ImageJ software (NIH, http://rsb.info.nih.gov/ij/), and insertion events were registered and analyzed manually. Total events per minute per unit surface area were taken as the frequency of insertion and were normalized to the control group as $100 \%$. Images rendered along the $y-t$ axis were generated in Image $\mathrm{J}$ by rotating the original $x-y-t$ stack $90^{\circ}$ along the $y$-axis, and the maximum intensity of each $x$ line was projected onto a single pixel of the $y$-axis using the maximum-intensity projection algorithm in ImageJ.

For Figure $1 C$, the image of an MSN expressing pH-DRD2 was first acquired using a Plan Neo-Fluo $40 \times$ oil objective lens (NA, 1.30) and then acquired under the TIRF imaging mode through an Alpha Plan-Apo $100 \times$ oil objective lens (NA, 1.46); the latter results in a reduced field of view but increased spatial resolution (160 nm per pixel when using the $40 \times, 1.30$ NA objective lens, and $64 \mathrm{~nm}$ per pixel when using the $100 \times$, 1.46 NA objective lens).

To quantify the single GFP fluorescence level, recombinant GFP (Clontech) was diluted to a concentration of $100 \mathrm{ng} / \mathrm{ml}$ and imaged under acquisition settings identical to those used for $\mathrm{pH}$-DRD2 (laser power, TIRF angle, objective, camera exposure, and gain setting). Single GFP fluorescent spots were identified manually based on their single-step photobleach characteristics. The primary peak on the histogram plot of single GFP fluorescence was then fitted to a Gaussian function using Igor Pro (WaveMetrics).

For Figure 3, neurons were pretreated with $20 \mu \mathrm{M}$ bicuculline (Bicu) for $1 \mathrm{~h}$ before recording was started, and neurons were recorded in ACSF buffer containing $20 \mu \mathrm{M}$ bicuculline. For DA stimulation, MSNs were pretreated with $1 \mu \mathrm{M}$ of DA for $20 \mathrm{~min}$. DA was then washed out, and recording was performed in ACSF. In experiments using dopamine $\mathrm{D}_{1}$ receptor (DRD1) antagonist SCH39166 (SCH; $\mathrm{C}_{19} \mathrm{H}_{20} \mathrm{CINO} . \mathrm{HBr}$ ), neurons were pretreated with $50 \mathrm{~nm}$ of SCH39166 and $1 \mu \mathrm{M}$ of DA for $20 \mathrm{~min}$, and neurons were recorded in ACSF buffer containing $50 \mathrm{~nm}$ of SCH39166. In experiments using DRD2 antagonist L741626 (L74; $\mathrm{C}_{20} \mathrm{H}_{21} \mathrm{CIN}_{2} \mathrm{O}$ ), neurons were pretreated with $1 \mu \mathrm{M}$ of L741626 and $1 \mu \mathrm{M}$ of DA for $20 \mathrm{~min}$, and neurons were recorded in ACSF buffer containing $1 \mu \mathrm{M}$ of L741626.

For Figure 5 photobleaching experiments, neurons were first identified on stage. pH-DRD2 molecules exposed on the surface were photobleached under the TIRFM condition for $1 \mathrm{~min}$, which resulted in elimination of most surface pH-DRD2 fluorescence. Neurons were then stimulated with dopamine, and recording was performed immediately following the onset of dopamine stimulation or $20 \mathrm{~min}$ after dopamine stimulation.

For all live-imaging experiments, neurons were used for imaging experiments within $30 \mathrm{~min}$ after they were removed from the incubator and incubated in ACSF.

Immunocytochemistry. Medium spiny neurons were transfected with $\mathrm{pH}-\mathrm{DRD} 2$ for $48 \mathrm{~h}$. Neurons were washed with ACSF and incubated with polyclonal rabbit anti-GFP antibody at a dilution of 1:200 (a gift from Dr. Richard L. Huganir, Department of Neuroscience, Johns Hopkins University School of Medicine/HHMI) for $1 \mathrm{~h}$ at $4^{\circ} \mathrm{C}$ to label pH-DRD2 on the surface. Neurons were then fixed with Parafix (4\% sucrose, $4 \%$ paraformaldehyde in PBS, $\mathrm{pH} 7.4$ ), permeabilized with $0.25 \%$ Triton $\mathrm{X}-100$ in PBS, blocked with $10 \%$ normal donkey serum in $\mathrm{PBS}$ at $37^{\circ} \mathrm{C}$ for $1 \mathrm{~h}$, and subsequently stained with a monoclonal GFP antibody at a dilution of 1:100 (a gift from Dr. Richard L. Huganir) to stain for total pH-DRD2 to amplify green fluorescent signal from superecliptic pHluorin at neutral $\mathrm{pH}$. Neurons were then washed four times and subsequently stained with Rhodamine Red-X donkey anti-Rabbit IgG (H+L; 1:100 dilution) and Alexa Fluor 488 donkey anti-mouse IgG (H+L; 1:200 dilution) secondary antibodies (Jackson ImmunoResearch). Neurons were then washed five times and mounted on slides. Images were acquired on an inverted Zeiss fluorescent microscope using a $40 \times$ objective (NA, 1.30) and a $2.5 \times$ relay lens between the microscope and the camera. Fluorescent intensities were quantified using Image (NIH, http://rsb.info.nih. gov/ij/). The ratio between surface to total of pH-DRD2 signal was normalized to the control group ( $\mathrm{pH}-\mathrm{DRD} 2$ without DA stimulation) as $100 \%$.

Statistics. All statistical tests were performed using Prism (GraphPad Software). All values were expressed as mean + SEM. The nonparametric Mann-Whitney test was used to compare statistical differences between any two groups. A value of $p<0.05$ was taken as the statistically significant difference.

\section{Results}

\section{Visualization of individual vesicular DRD2 insertion events}

There are two DRD2 splice variants: a long isoform (DRD2L) and a short isoform (DRD2S). Previous studies have suggested that DRD2L mediates postsynaptic DRD2 function, while DRD2S is considered to be an autoreceptor on dopamine neurons (Mack et al., 1991; Neve et al., 1991; Khan et al., 1998; Usiello et al., 2000; Rouge-Pont et al., 2002; Xu et al., 2002; Fetsko et al., 2003; Lindgren et al., 2003; Holmes et al., 2004). Our current study focuses on regulation of DRD2 trafficking in striatal MSNs, which are postsynaptic to dopamine neurons. We therefore focus our study primarily on DRD2L. To visualize vesicular insertion events of DRD2L to the PM, we first labeled the N terminus of DRD2L with a pH-sensitive GFP, the superecliptic pHluorin (Miesenbock et al., 1998), and generated a pH-DRD2L construct (pRK5-PHDRD2L) using a strategy similar to that applied to the $\beta 2$ adrenergic receptor (Yudowski et al., 2006) (Fig. 1A). The response of this $\mathrm{pH}-\mathrm{DRD} 2 \mathrm{~L}$ construct to three physiological concentrations of DA was similar to that of the nontagged version of DRD2L (Fig. $1 B$ ), demonstrating that tagging the $\mathrm{N}$ terminus of DRD2L with superecliptic pHluorin does not interfere with its function. We next cultured striatal MSNs from embryonic mice and transfected with pKR5-pH-DRD2L for our imaging experiments (Fig. $1 C$, left). Using TIRFM we were able to visualize the sudden appearance of $\mathrm{pH}-\mathrm{DRD} 2 \mathrm{~L}$ clusters on both the somatic and dendritic surfaces of MSNs (Fig. 1C, middle, right). Untransfected MSNs or MSNs transfected with nontagged DRD2L displayed no fluorescence or visible insertion (data not shown). We determined that the dynamic $\mathrm{pH}-\mathrm{DRD} 2 \mathrm{~L}$ clusters represent insertion events of pH-DRD2L from intracellular vesicles into the PM, rather than clustering of surface DRD2L molecules, by showing that appearance of these clusters could be abolished by coexpression of pH-DRD2L with tetanus toxin light chain (TeNTLC; insertion frequency, control, $100 \pm 11 \%, n=22$ neurons; TeNTLC, $27 \pm 7 \%, n=14$ neurons; $p<0.0001$, Mann-Whitney test) (Fig. $1 D$ ), which blocks vesicle insertion into the PM (Lin et al., 2009).

To quantify the number of DRD2L molecules in individual insertion events, we first quantified the fluorescence level of a single recombinant GFP molecule. Identification of a single GFP molecule is based on the criterion that photobleaching of a single GFP molecule is a single-step process (Fig. $1 E$ ). Under our imaging conditions, the fluorescence intensity of a single GFP molecule was $34,322 \pm 343$ arbitrary units (A.U.), from quantification of 813 single GFP molecules (Fig. $1 F$ ). On average, the total peak fluorescence intensity of pH-DRD2L insertion was 1,020,932 \pm 32,069 A.U. ( $n=747$ individual insertion events from 135 neurons); therefore, each $\mathrm{pH}-\mathrm{DRD} 2 \mathrm{~L}$ insertion event, on average, comprised $30 \pm 1$ DRD2L molecules (Fig. 1G). Insertion events appeared as the rapid appearance of $\mathrm{pH}-\mathrm{DRD} 2 \mathrm{~L}$ clusters that then dispersed within seconds. We observed at least two types of 
A

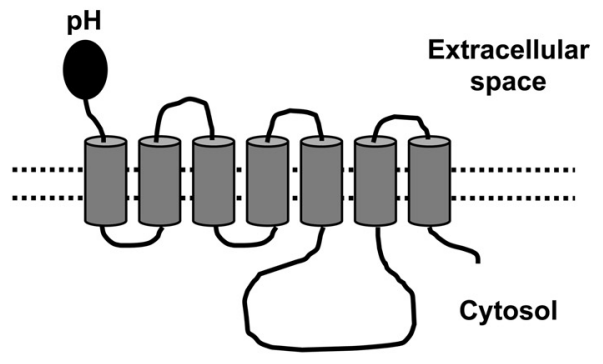

C

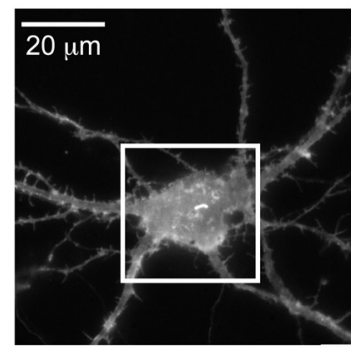

E
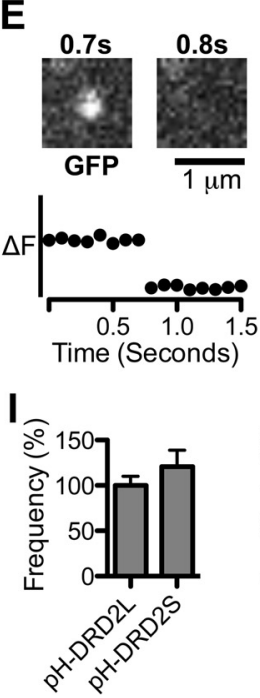
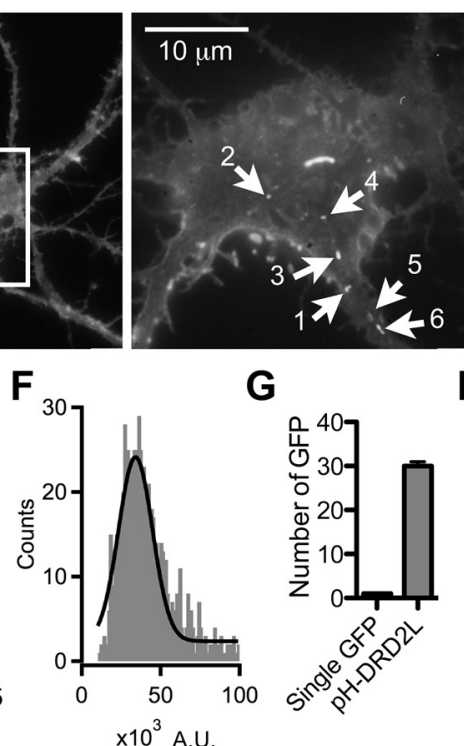

G

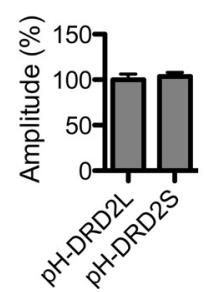

B

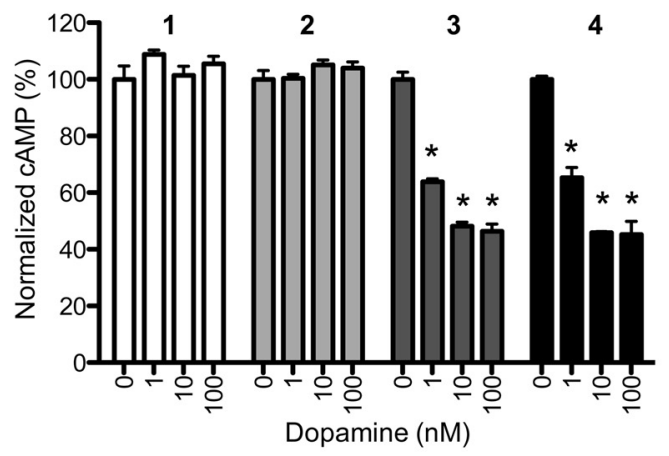

D
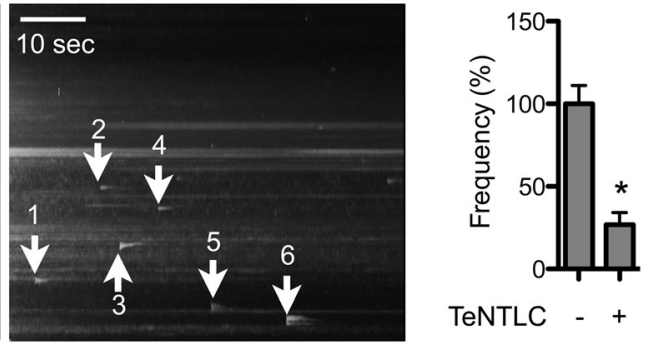

TeNTLC - +

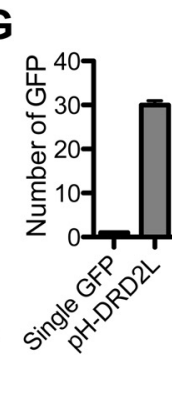

J

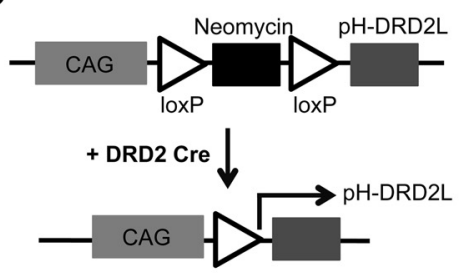

H

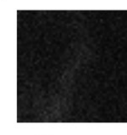

0 sec

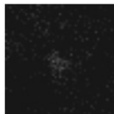

$0 \mathrm{sec}$

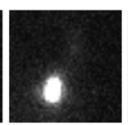

$1.0 \mathrm{sec}$
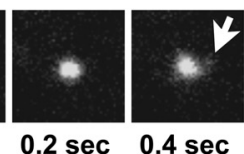

$\mathrm{K}$

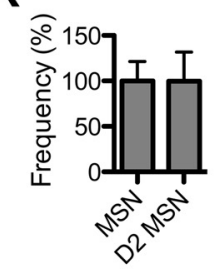

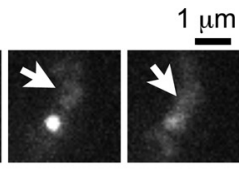

$4.0 \mathrm{sec} 5.0 \mathrm{sec}$

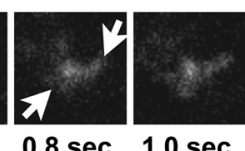

Figure 1. Visualization of pH-DRD2 insertion in cultured MSNs. $\boldsymbol{A}$, Schematic drawing of the pH-DRD2 construct. $\boldsymbol{B}$, cAMP accumulation assay to determine DRD2 function in HEK 293 cells: untransfected cells (1), vector-transfected cells (2), DRD2 (nontagged) transfected cells (3), and pH-DRD2 transfected cells (4). Activation of DRD2 inhibits cAMP accumulation. Untransfected and vector-transfected cells do not respond to DA stimulation, while DRD2 (nontagged) and pH-DRD2 display similar sensitivities to DA at three different concentrations: 1,10 , and 100 nm. C, Left, pH-DRD2L in a cultured MSN visualized under epifluorescent mode using a $40 \times, 1.30$ NA objective. The white square in the middle represents the field of view under TIRF imaging mode using a $100 \times, 1.46$ NA objective, as seen in the middle. Middle, Maximum-intensity projection image of pH-DRD2L fluorescence of the same cultured MSN visualized under TIRF imaging mode; white spots with arrowheads represent individual pH-DRD2L insertion events as visualized under TIRFM. Right, $y$ - $t$ maximum-intensity projection image of pH-DRD2L fluorescence in the same cultured MSN visualized under TIRF imaging mode; arrowheads indicate individual pH-DRD2 insertion events as indicated in the middle panel. $D, 0$ uantification of pH-DRD2L insertion frequency with or without TeNTLC. $\boldsymbol{E}$, The two images show a single GFP fluorescent spot before (left) and after (right) the single-step photobleaching; the plot underneath the images shows fluorescence changes over time at this fluorescent spot. Single-step photobleaching occurs between 0.7 and $0.8 \mathrm{~s}$. F, Fitting the primary peak of single GFP fluorescence intensity to a Gaussian function results in a Gaussian profile with the center of the Gaussian function representing a single GFP fluorescence level. G, By normalizing the average total fluorescence level of pH-DRD2 to single GFP fluorescence, the number of pH-DRD2L molecules in individual insertion events can be derived. $\boldsymbol{H}$, Two representative insertion events of pH-DRD2 followed by lateral diffusion. The arrows indicate diffusion of pH-DRD2L from the initial insertion site. I, Comparison of pH-DRD2L and pH-DRD2S in MSNs. J, Experimental design for examination of pH-DRD2L insertion properties in D2-type MSNs. In pCALNL-pH-DRD2L, the translation of pH-DRD2L is prevented by a floxed neomycin cassette. Transfecting this pCALNL-pH-DRD2L construct into MSNs cultured from DRD2 Cre embryos will result in specific expression of pH-DRD2L in $\mathrm{D}_{2}$-type MSNs. $K$, Comparison of pH-DRD2L insertion between MSNs and $\mathrm{D}_{2}$-type MSNs. MSN, pH-DRD2L insertion without discrimination of $\mathrm{D}_{1}$-type versus $\mathrm{D}_{2}$-type MSNs, using pRK5-pH-DRD2L construct; $\mathrm{D}_{2}$ MSN, pH-DRD2L insertion in $\mathrm{D}_{2}$-type MSNs using pCALNL-pH-DRD2L construct. Asterisks denote statistical significance compared to the control group.

diffusion following insertion: (1) a relatively slow diffusion, occurring primarily in one direction (Fig. $1 H$, top), and (2) a more rapid diffusion, with $\mathrm{pH}-\mathrm{DRD} 2 \mathrm{~L}$ diffusing in different directions immediately following insertion (Fig. $1 \mathrm{H}$, bottom).

$\mathrm{pH}-\mathrm{DRD} 2 \mathrm{~S}$ showed insertion frequencies and amplitudes similar to those of $\mathrm{pH}-\mathrm{DRD} 2 \mathrm{~L}$ (insertion frequency, DRD2L, $100 \pm$
$10 \%, n=30$ neurons; DRD2S, $121 \pm 18 \%, n=32$ neurons; $p=$ 0.9047, Mann-Whitney test; insertion amplitude, DRD2L, $100 \pm$ $6 \%, n=182$ events from 30 neurons; DRD2S, $104 \pm 5 \%, n=217$ events from 32 neurons; $p=0.1085$, Mann-Whitney test) (Fig. $1 I$ ). Based on the absence of difference between the insertion of the two DRD2 isoforms and the suggested postsynaptic role of DRD2L, we 
focus our current studies on DRD2L. But we do not exclude that similar mechanisms can also drive DRD2S trafficking.

In the striatum, MSNs can be categorized as $\mathrm{D}_{1}$ - or $\mathrm{D}_{2}$-type MSNs. Similarly, both $\mathrm{D}_{1}$ - and $\mathrm{D}_{2}$-type MSNs exist in our primary cultured system, and have equal chance to be transfected with pRK5-pHDRD2L for our imaging experiments (data not shown). It was not known whether $\mathrm{D}_{1}$ - and $\mathrm{D}_{2}$-type MSNs have different DRD2 insertion properties. We therefore designed an experiment allowing us to specifically label $\mathrm{D}_{2}$-type MSNs and examine $\mathrm{pH}-\mathrm{DRD} 2 \mathrm{~L}$ insertion only in $\mathrm{D}_{2}$-type MSNs. To accomplish this, we replaced the DsRed cDNA on the pCALNL-DsRed vector (Matsuda and Cepko, 2007) with pH-DRD2L, thereby generating a pCALNL-pH-DRD2L expression construct with a neomycin cassette with two loxP sites in front of the pH-DRD2L cDNA (Fig. 1J). With this design, the translation of $\mathrm{pH}-\mathrm{DRD} 2 \mathrm{~L}$ is prevented by the translation of neomycin. However, in neurons expressing Cre, the neomycin cassette is removed via Cremediated recombination, allowing translation of pH-DRD2L. We next obtained the DRD2 Cre mouse strain, in which $\mathrm{D}_{2}-$ type MSNs are labeled with Cre and then cultured neurons from heterozygous DRD2 Cre embryos (see Materials and Methods) to ensure that all of the $\mathrm{D}_{2}$-type MSNs were labeled with Cre. We separated our culture into two groups. The first group of neurons were transfected with the regular $\mathrm{pH}$ DRD2L construct ( $\mathrm{pRK} 5-\mathrm{pH}-\mathrm{DRD} 2 \mathrm{~L}$ ), which results in expression of $\mathrm{pH}$-DRD2L in both $\mathrm{D}_{1}$ - and $\mathrm{D}_{2}$-type MSNs (Fig. $1 \mathrm{~K}$, MSN), similar to most of our imaging experiments. The second group of neurons were transfected with the pCALNL-pHDRD2L construct, allowing expression of $\mathrm{pH}-\mathrm{DRD} 2 \mathrm{~L}$ specifically in $\mathrm{D}_{2}$-type MSNs (Fig. $1 \mathrm{~K}, \mathrm{D}_{2} \mathrm{MSN}$ ). Using this strategy, the insertion of $\mathrm{pH}$-DRD2L in $\mathrm{D}_{2}$-type MSNs showed insertion frequencies and amplitudes similar to those of the mixed $D_{1}$ - and $D_{2}$-type MSNs (insertion frequency, MSN, $100 \pm 21 \%, n=15$ neurons; $\mathrm{D}_{2} \mathrm{MSN}$, $100 \pm 32 \%, n=17$ neurons; $p=0.5205$, Mann-Whitney test; insertion amplitude, MSN, $100 \pm 8 \%, n=60$ events from 15 neurons; $\mathrm{D}_{2}$ MSN, $115 \pm 10 \%, n=63$ events from 17 neurons; $p=0.1426$, Mann-Whitney test) (Fig. $1 \mathrm{~K}$ ). Therefore, we concluded that results obtained from unidentified (i.e., mixed $\mathrm{D}_{1}$ - and $\mathrm{D}_{2}$-type) MSNs are similar to those from $\mathrm{D}_{2}$-type MSNs. Given that identifying positive transfected $\mathrm{D}_{2}$-type MSNs is very inefficient, we therefore performed most of our imaging experiments in mixed MSNs.

\section{Observed DRD2L insertion represents constitutive DRD2L recycling}

The observed DRD2L insertion could originate either from forward trafficking in the biosynthetic pathway or from the endosomal recycling pathways. Insertion from the biosynthetic pathway does not rely on receptor endocytosis, whereas endosomal recycling depends on receptor endocytosis from the PM. DRD2 endocytosis depends on clathrin and arrestin (Kim et al.,
2001; Kabbani et al., 2004; Paspalas et al., 2006). Hypertonic sucrose, which inhibits clathrin-dependent endocytosis, significantly reduced the $\mathrm{pH}-\mathrm{DRD} 2 \mathrm{~L}$ insertion frequency (without sucrose, $100 \pm 15 \%, n=33$ neurons; with sucrose, $34 \pm 9 \%, n=$ 16 neurons; $p=0.0003$, Mann-Whitney test), but did not affect the amplitude of $\mathrm{pH}-\mathrm{DRD} 2 \mathrm{~L}$ insertion (without sucrose, $100 \pm$ $11 \%, n=211$ events from 33 neurons; with sucrose, $102 \pm 23 \%$, $n=32$ events from 16 neurons; $p=0.9451$, Mann-Whitney test) (Fig. 2A). This result suggests that observed pH-DRD2L insertion represents endosomal recycling of DRD2L, and that inhibiting clathrin-dependent DRD2L endocytosis reduces the total amount of DRD2L available for recycling, but does not affect the loading of DRD2L into each vesicle for recycling. We next generated a $\mathrm{pH}$-DRD2L I ${ }^{212} \mathrm{Y}^{213} \mathrm{I}^{214} \mathrm{~V}^{215}-\mathrm{A}^{212} \mathrm{~A}^{213} \mathrm{~A}^{214} \mathrm{~A}^{215}$ mutant that abolished arrestin binding and DRD2 endocytosis but left DRD2 signaling through the G-protein pathways intact (Lan et al., 2009). This pH-DRD2L mutant also displayed significantly reduced $\mathrm{pH}-\mathrm{DRD} 2 \mathrm{~L}$ insertion frequency $(\mathrm{pH}-\mathrm{DRD} 2 \mathrm{~L}, 100 \pm$ $19 \%, n=27$ neurons; $\mathrm{pH}-\mathrm{DRD} 2 \mathrm{~L}$ IYIV_4A, $23 \pm 9 \%, n=16$; $p=0.0017$, Mann-Whitney test), whereas the amplitude of insertion was unchanged ( $\mathrm{pH}-\mathrm{DRD} 2 \mathrm{~L}, 100 \pm 14 \%, n=80$ events from 27 neurons; pH-DRD2L IYIV_4A, $71 \pm 22 \%, n=10$ events from 16 neurons; $p=0.1675$, Mann-Whitney test) (Fig. $2 B$ ). This observation further suggests that the observed $\mathrm{pH}-\mathrm{DRD} 2 \mathrm{~L}$ insertion represents endosomal recycling of DRD2L. Different endosomal recycling pathways are regulated by different small Rab GTPases. For example, Rab4 is involved in fast recycling 
A

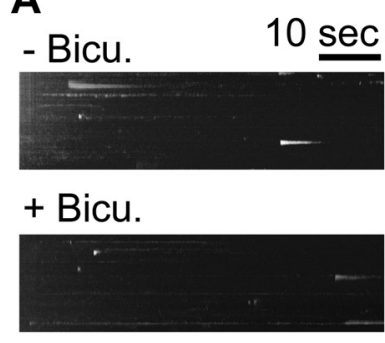

B

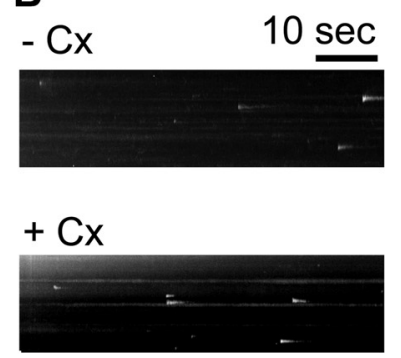

C

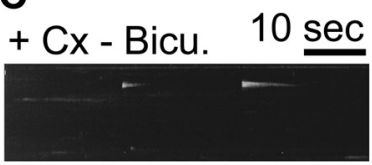

$+C x+$ Bicu.

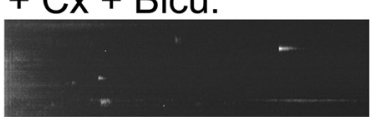

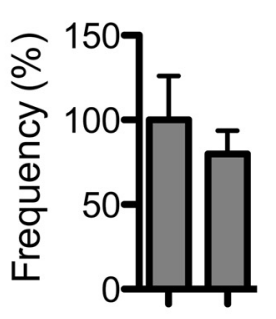

Bicu. - +

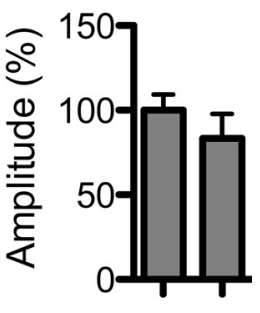

Bicu. - +

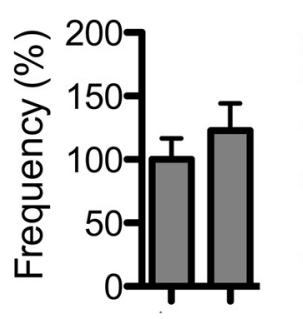

Cx - +

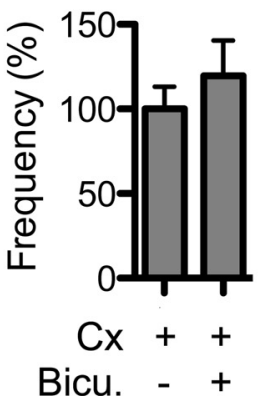

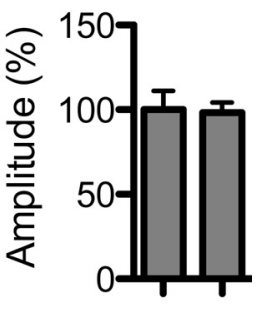

Cx - +

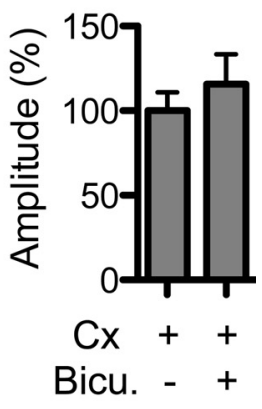

Figure 3. Glutamatergic or GABAergic activity does not affect pH-DRD2 insertion. Left, $y$ - $t$ maximum-intensity projection images of pH-DRD2 insertion events in each experimental group. $A$, Inhibition of GABAergic activity does not affect pH-DRD2 insertion in MSN. Bicu., $20 \mu$ m bicuculline; - Bicu., pH-DRD2 insertion in the absence of bicuculline; + Bicu., pH-DRD2 insertion in the presence of bicuculline. $B$, Coculture of cortical neurons with MSN does not affect pH-DRD2 insertion. C, Inhibition of GABAergic activity in coculture of MSN and $\mathrm{Cx}$ does not affect pH-DRD2 insertion.

from sorting endosomes to the PM, while Rab11 is involved in slow recycling from recycling endosomes to the PM (Stenmark, 2009). To further determine whether the observed $\mathrm{pH}-\mathrm{DRD} 2 \mathrm{~L}$ insertion originated from endosomal recycling pathways, we coexpressed dominant-negative mutants of either Rab4 or Rab11 with $\mathrm{pH}-\mathrm{DRD} 2 \mathrm{~L}$ and recorded $\mathrm{pH}-\mathrm{DRD} 2 \mathrm{~L}$ insertion. Our results showed that Rab4DN significantly reduced both $\mathrm{pH}-\mathrm{DRD} 2 \mathrm{~L}$ insertion frequency ( $\mathrm{pH}-\mathrm{DRD} 2 \mathrm{~L}, 100 \pm 7 \%, n=65$ neurons; pH-DRD2L+Rab4DN, $45 \pm 6 \%, n=36$ neurons; $p<0.0001$ compared to $\mathrm{pH}-\mathrm{DRD} 2 \mathrm{~L}$, Mann-Whitney test) and amplitude (pH-DRD2L, $100 \pm 4 \%, n=474$ events from 65 neurons; $\mathrm{pH}$ DRD2L+Rab4DN, $68 \pm 7 \%, n=105$ events from 36 neurons; $p<$ 0.0001 compared to $\mathrm{pH}-\mathrm{DRD} 2 \mathrm{~L}$, Mann-Whitney test) under basal conditions (Fig. 2C), suggesting that the observed DRD2L insertion represents its recycling from sorting endosomes to the PM. Interestingly, Rab11DN did not affect pH-DRD2L insertion frequency ( $\mathrm{pH}-\mathrm{DRD} 2 \mathrm{~L}+\mathrm{Rab} 11 \mathrm{DN}, 84 \pm 8 \%, n=39$ neurons; $p=0.1131$ compared to $\mathrm{pH}-\mathrm{DRD} 2 \mathrm{~L}$, Mann-Whitney test), but significantly increased $\mathrm{pH}-\mathrm{DRD} 2 \mathrm{~L}$ insertion amplitude $(\mathrm{pH}-$ DRD2L+Rab11DN, $145 \pm 11 \%, n=211$ events from 39 neurons; $p=0.0002$ compared to $\mathrm{pH}-\mathrm{DRD} 2 \mathrm{~L}$, Mann-Whitney test) (Fig.

$2 C)$. This result suggests that under basal conditions, DRD2L recycling does not originate from Rab11-dependent recycling endosomes. Together, these results demonstrate that under basal conditions, the observed pH-DRD2L insertion represents constitutive recycling of DRD2L from sorting endosomes to the PM.

\section{Glutamatergic or GABAergic activity does not affect DRD2 insertion}

Glutamatergic and GABAergic activities regulate the trafficking of glutamate receptors and $\mathrm{GABA}_{\mathrm{A}}$ receptors in neurons and have been shown to play important roles in regulating the function of these receptors in glutamatergic and GABAergic synapses (Shepherd and Huganir, 2007; Luscher et al., 2011). However, to date it is not known whether these neuronal activities affect DRD2 trafficking in MSNs. In the striatum, $>90 \%$ of neurons are GABAergic MSNs. They receive GABAergic innervation from local inhibitory interneurons as well as from other MSNs, and glutamatergic innervation from the cortex and other brain regions. To determine whether GABAergic or glutamatergic activity in MSNs affects DRD2L insertion, we manipulated GABAergic and glutamatergic activities in our cultures and recorded $\mathrm{pH}-\mathrm{DRD} 2 \mathrm{~L}$ insertion in $\mathrm{MSN}$. The $\mathrm{GABA}_{\mathrm{A}}$ receptor antagonist bicuculline $(20 \mu \mathrm{M})$ affected neither the frequency nor the amplitude of DRD2L insertion in MSNs [insertion frequency, without Bicu (-Bicu), $100 \pm$ $26 \%, n=21$ neurons; with Bicu ( + Bicu), $80 \pm 14 \%, n=16$ neurons; $p=0.5195$, Mann-Whitney test; insertion amplitude, - Bicu, $100 \pm 9 \%, n=111$ events from 21 neurons; $+\mathrm{Bicu}, 83 \pm 14 \%, n=87$ events from 16 neurons; $p=0.2640$, Mann-Whitney test] (Fig. $3 A$ ), suggesting that DRD2L insertion is not regulated by GABAergic input to MSNs. To determine whether excitatory glutamatergic activity in MSNs affects DRD2L insertion, we cocultured MSNs with cortical neurons $(\mathrm{Cx})$, which provide glutamatergic innervation to MSNs (Sun et al., 2008). We then compared DRD2 insertion in MSNs in the presence or absence of cortical neurons. Our results showed that coculture of MSNs with cortical neurons affected neither the frequency nor the amplitude of DRD2L insertion in MSNs [insertion frequency, without $\mathrm{Cx}$ coculture $(-\mathrm{Cx}), 100 \pm 17 \%, n=29$ neurons; with Cx coculture $(+\mathrm{Cx})$, $123 \pm 21 \%, n=25$ neurons; $p=0.5788$, Mann-Whitney test; insertion amplitude, $-\mathrm{Cx}, 100 \pm 11 \%, n=149$ events from 29 neurons; $+\mathrm{Cx}, 98 \pm 6 \%, n=175$ events from 25 neurons; $p=$ 0.0804, Mann-Whitney test] (Fig. 3B). Because our striatal culture contained a large number of GABAergic MSNs, which could potentially inhibit the activity of cortical neurons, we further increased glutamatergic activity in our cocultures by inhibiting GABAergic activity using bicuculline. Consistently, bicuculline treatment affected neither the frequency nor the amplitude of DRD2L insertion in MSNs cocultured with cortical neurons (in- 
sertion frequency, $+\mathrm{Cx}-\mathrm{Bicu}, 100 \pm$ $13 \%, n=21$ neurons; $+\mathrm{Cx}+\mathrm{Bicu}, 120 \pm$ $21 \%, n=20$ neurons; $p=0.8246$, MannWhitney test; insertion amplitude, +Cx - Bicu, $100 \pm 11 \%, n=98$ events from 21 neurons; $+\mathrm{Cx}+\mathrm{Bicu}, 116 \pm 18 \%, n=$ 119 events from 20 neurons; $p=0.6750$, Mann-Whitney test) (Fig. 3C). Together, these results demonstrate that glutamatergic and GABAergic inputs to MSNs do not affect DRD2L insertion to the PM and suggest that our striatal MSN culture preparation without cortical neuron coculture is a simple and excellent cellular model system for studying dynamic DRD2L trafficking.

\section{DA activity-dependent DRD2L insertion}

In the striatum, MSNs also receive dopaminergic innervation from the ventral tegmental area and substantia nigra pars compacta. To determine whether dopaminergic activity affects DRD2L insertion, we stimulated MSNs with $1 \mu \mathrm{M}$ DA for 20 min and then recorded $\mathrm{pH}-\mathrm{DRD} 2 \mathrm{~L}$ insertion using TIRFM. Our results showed that $20 \mathrm{~min}$ of DA stimulation significantly increased both the frequency and the amplitude of $\mathrm{pH}-\mathrm{DRD} 2 \mathrm{~L}$ insertion [insertion frequency, without DA stimulation ( $-\mathrm{DA}$ ), $100 \pm 9 \%, n=68$ neurons; with DA stimulation (+DA), $232 \pm 26 \%$, $n=70$ neurons; $p<0.0001$, Mann-Whitney test; insertion amplitude, -DA, $100 \pm 6 \%, n=357$ events from 68 neurons; $+\mathrm{DA}, 180 \pm 12 \%, n=649$ events from 70 neurons; $p<0.0001$, MannWhitney test] (Fig. 4A), suggesting that $\mathrm{DRD} 2 \mathrm{~L}$ insertion is regulated by DA activity. The increase in $\mathrm{pH}-\mathrm{DRD} 2 \mathrm{~L}$ insertion following DA stimulation is not affected by the DRD1 antagonist SCH39166 (insertion frequency, $-\mathrm{DA}, 100 \pm 15 \%, n=$ 18 neurons; +DA, $319 \pm 35 \%, n=17$ neurons; $p<0.0001$ compared to $-\mathrm{DA}$; $+\mathrm{DA}+\mathrm{SCH} 39166,295 \pm 30 \%, n=16$ neurons; $p<0.0001$ compared to $-\mathrm{DA}$, $p=0.7322$ compared to $+\mathrm{DA}$; insertion amplitude, $-\mathrm{DA}, 100 \pm 6 \%, n=108$ events from 18 neurons; +DA, $137 \pm$ $6 \%, n=300$ events from 17 neurons; $p=0.0003$ compared to $-\mathrm{DA} ;+\mathrm{DA}$ $+\mathrm{SCH} 39166,138 \pm 7 \%, n=297$ events from 16 neurons; $p=0.0007$ compared to $-\mathrm{DA}, p=0.7666$ compared to $+\mathrm{DA}$, Mann-Whitney test) (Fig. $4 B$ ), but is abolished by DRD2 antagonist L741626 (insertion frequency, $-\mathrm{DA}, 100 \pm 13 \%$, $n=15$ neurons; $+\mathrm{DA}, 227 \pm 36 \%, n=13$ neurons; $p=0.0011$ compared to $-\mathrm{DA}$; $+\mathrm{DA}+\mathrm{L} 741626,100 \pm 19 \%, n=18 \mathrm{neu}-$ rons; $p=0.6255$ compared to $-\mathrm{DA}, p=$
A

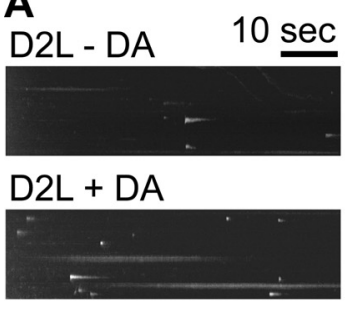

B
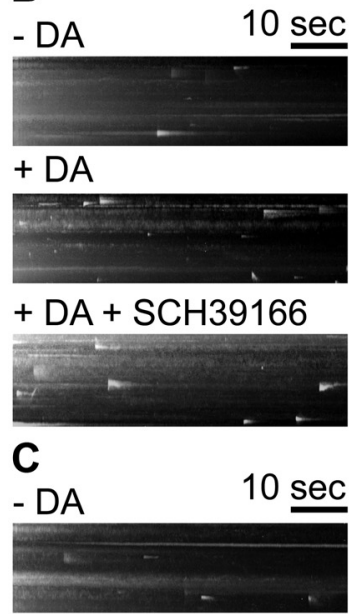

$+\mathrm{DA}$

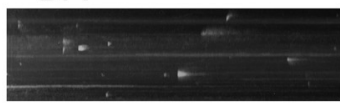

$+\mathrm{DA}+\mathrm{L} 741626$

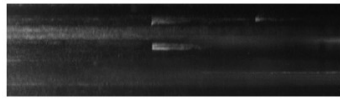

D

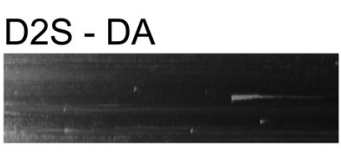

$D 2 S+D A$

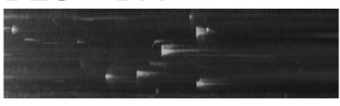

E
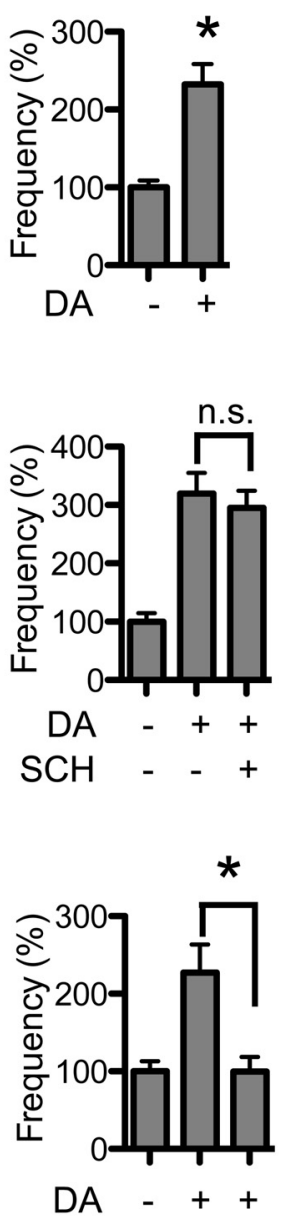

L74 - -+

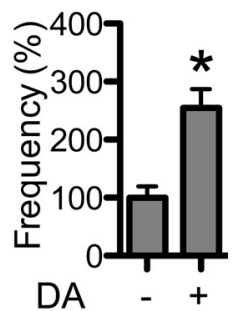

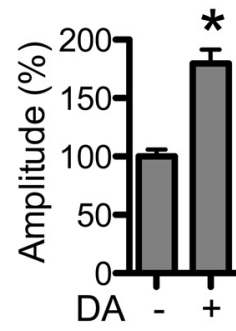
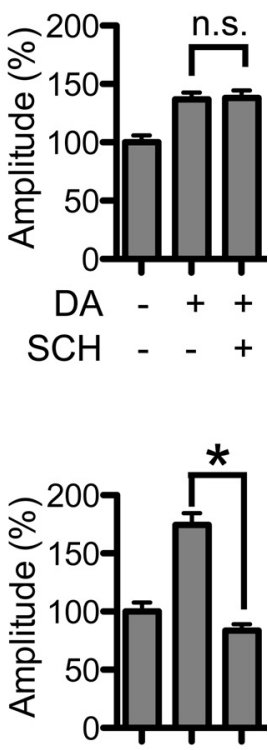

DA - + +

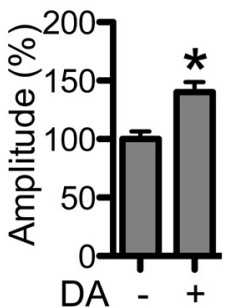

L74 - - +

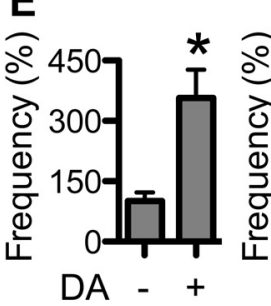

MSN

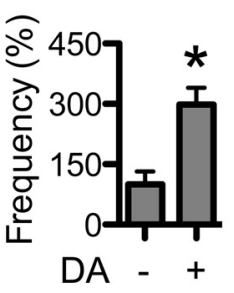

D2 MSN

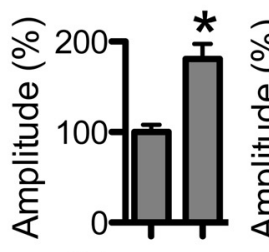

DA - +

MSN

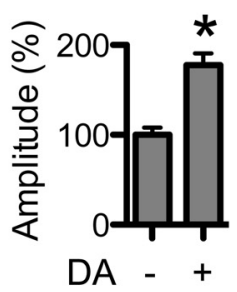

D2 MSN
Figure 4. DA activity-dependent pH-DRD2 insertion. Left, $y-t$ maximum-intensity projection images of pH-DRD2 insertion events in each experimental group. $A$, Twenty minute treatment with $1 \mu \mathrm{mDA}$ enhances the frequency and amplitude of pH-DRD2 insertion in MSN. $\boldsymbol{B}$, Dopamine $\mathrm{D}_{1}$ receptor antagonist $S C H$ does not affect DA activity-dependent pH-DRD2 insertion. $C$, DRD2 antagonist L74 abolishes DA activity-dependent pH-DRD2 insertion. $\boldsymbol{D}$, pH-DRD2S also showed DA activity-dependent insertion. $\boldsymbol{E}$, Comparison of DA activitydependent $\mathrm{pH}-\mathrm{DRD} 2 \mathrm{~L}$ insertion in MSNs versus $\mathrm{D}_{2}$-type MSNs. MSN, $\mathrm{pH}-\mathrm{DRD2L}$ insertion in MSNs without discrimination of $\mathrm{D}_{1}$ - versus $D_{2}$-type MSNs; $D_{2}$ MSNs, pH-DRD2L insertion in DRD2 Cre-labeled D -type MSNs. -DA, pH-DRD2 insertion in the absence of DA stimulation; +DA, pH-DRD2 insertion following DA stimulation; +DA + SCH39166, pH-DRD2L with DA stimulation in the presence of DRD1 antagonist SCH39166; +DA + L741626, pH-DRD2L with DA stimulation in the presence of DRD2 antagonist L741626. Asterisks denote statistical significance compared to the control group. n.S., Not significant. 
A

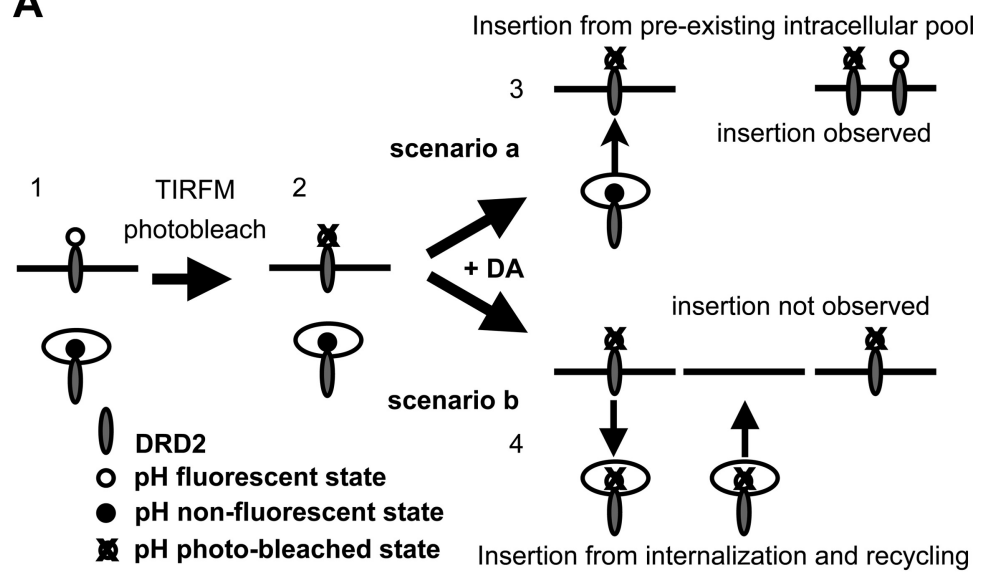

B

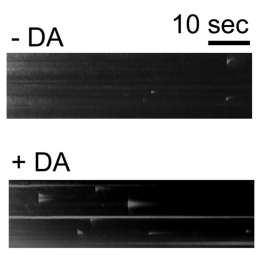

C

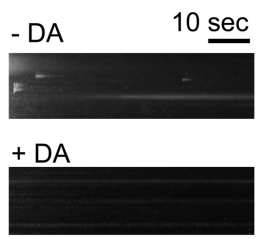

D

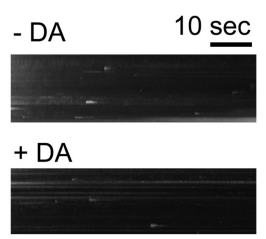

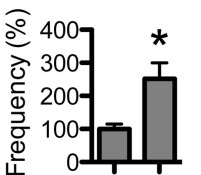

DA - +

DA 20 min, no bleach

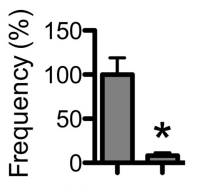

$\mathrm{DA}-+$
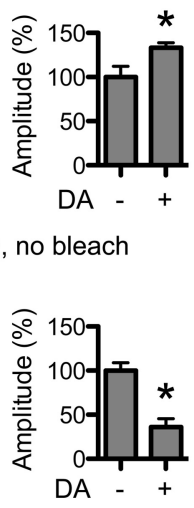

DA 20 min, bleach
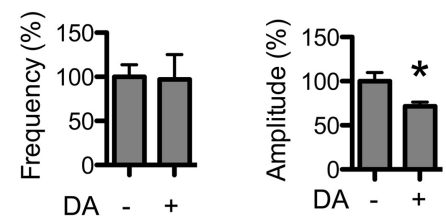

DA 0 min, bleach

Figure 5. DA activity facilitates recycling of internalized DRD2L. $A$, Rationale and design for the photobleaching experiments: (1) Before photobleaching, PH-DRD2L on the PM is fluorescent, and pH-DRD2L in intracellular pools is nonfluorescent. (2) Photobleach is performed in TIRFM mode; consequently, $\mathrm{pH}-\mathrm{DRD} 2 \mathrm{~L}$ on the PM is photobleached, but due to the nonfluorescent nature of intracellular pH-DRD2L and photobleach in TIRFM mode, pH-DRD2L in intracellular pools is protected from photobleach. (3) Scenario a, If DA stimulation enhances the insertion of pH-DRD2L that originated from intracellular pools, then DA stimulation should still be able to increase pH-DRD2L insertion after photobleaching, as intracellular pools of pH-DRD2L would be protected from photobleach. (4) Scenario b, If DA stimulation enhances the recycling of internalized pH-DRD2L, then photobleaching should result in a photobleached pHluorin attached to the recycled DRD2L; hence, insertion of DRD2L should be undetectable to our imaging system, and we would expect to observe a significant reduction in DRD2L insertion. $\boldsymbol{B}$, Without photobleaching, 20 min of DA stimulation consistently enhances the frequency and amplitude of $\mathrm{pH}-\mathrm{DRD} 2 \mathrm{~L}$ insertion. C, Following photobleaching, the same protocol of 20 min of DA stimulation almost completely abolishes $\mathrm{pH}-\mathrm{DRD} 2 \mathrm{~L}$ insertion, as predicted by scenario $\mathrm{b}$, suggesting that following DA stimulation, the observed pH-DRD2L insertion originates primarily from recycling of endocytosed DRD2L. D, Following photobleaching, and immediately after the onset of DA stimulation, $\mathrm{pH}-\mathrm{DRD} 2 \mathrm{~L}$ insertion is clearly visible but the frequency is not increased, and the amplitude is slightly reduced. Asterisks denote statistical significance compared to the control group.

0.0017 compared to + DA; insertion amplitude, $-\mathrm{DA}, 100 \pm 8 \%$, $n=100$ events from 15 neurons; $+\mathrm{DA}, 175 \pm 10 \%, n=149$ events from 13 neurons; $p<0.0001$ compared to $-\mathrm{DA} ;+\mathrm{DA}$ $+\mathrm{L} 741626,84 \pm 5 \%, n=98$ events from 18 neurons; $p=0.1451$ compared to $-\mathrm{DA}, p<0.0001$ compared to +DA, Mann-Whitney test) (Fig. 4C). Together, these results demonstrate that the increase in $\mathrm{pH}-\mathrm{DRD} 2 \mathrm{~L}$ insertion in response to DA stimulation requires activation of DRD2 but not of DRD1.

DA stimulation also increased both the frequency and amplitude of $\mathrm{pH}-\mathrm{DRD} 2 \mathrm{~S}$ insertion in MSNs (insertion frequency,
$-\mathrm{DA}, 100 \pm 19 \%, n=20 ;+\mathrm{DA}: 255 \pm$ $33 \%, n=16 ; p=0.0003$, Mann-Whitney test; insertion amplitude, $-\mathrm{DA}, 100 \pm$ $7 \%, n=161$ events from 20 neurons; + DA, $140 \pm 8 \%, n=250$ events from 16 neurons; $p=0.0003$, Mann-Whitney test) (Fig. 4D), suggesting that in MSNs, DRD2S insertion is likely subjected to regulatory mechanisms similar to those of DRD2L. We next performed experiments to determine whether results from mixed $\mathrm{D}_{1^{-}}$and $\mathrm{D}_{2}$-type $\mathrm{MSNs}$ are similar to those in $\mathrm{D}_{2}$-type MSNs, using strategy described in Fig. $1 \mathrm{~J}$. We found that in $\mathrm{D}_{2}$ type MSNs, pH-DRD2L displayed DA activity-dependent increases in insertion frequency and amplitude similar to those in mixed $\mathrm{D}_{1^{-}}$and $\mathrm{D}_{2}$-type MSNs (insertion frequency, MSN $-\mathrm{DA}, 100 \pm 21 \%, n=15$ neurons; MSN +DA, $357 \pm 70 \%, n=13$ neurons; $p=0.0037$, Mann-Whitney test; $\mathrm{D}_{2} \mathrm{MSN}-\mathrm{DA}, 100 \pm 32 \%, n=17$ neurons; $\mathrm{D}_{2} \mathrm{MSN}+\mathrm{DA}, 298 \pm 42 \%, n=13$ neurons; $p=0.0004$ compared to $\mathrm{D}_{2} \mathrm{MSN}-\mathrm{DA}, p=$ 0.6444 compared to MSN +DA; insertion amplitude, MSN -DA, $100 \pm 8 \%, n=60$ events from 16 neurons; MSN +DA, $181 \pm$ $17 \%, n=167$ events from 13 neurons; $p=$ 0.0036 compared to MSN - DA, MannWhitney test; $\mathrm{D}_{2} \mathrm{MSN}-\mathrm{DA}, 100 \pm 8 \%$, $n=63$ events from 17 neurons; $\mathrm{D}_{2} \mathrm{MSN}$ $+\mathrm{DA}, 178 \pm 13 \%, n=173$ events from 13 neurons; $p=0.0025$ compared to $\mathrm{D}_{2} \mathrm{MSN}$ $-\mathrm{DA}, p=0.8202$ compared to MSN $+\mathrm{DA}$, Mann-Whitney test) (Fig. $4 E$ ). These results further support the notion that result obtained from mixed $\mathrm{D}_{1}$-type and $\mathrm{D}_{2}$-type MSNs are similar to that from $\mathrm{D}_{2}$-type MSNs and suggests that the primary cultured MSN is an excellent in vitro model system for examination of DRD2L trafficking.

DA enhances DRD2L recycling through a distinct endosomal route

The DA activity-dependent increase in DRD2L insertion could originate either from preexisting intracellular pools of DRD2L or from recycling of DRD2L that was initially present on the PM and was endocytosed upon DA stimulation. To determine the origin of increased DRD2L insertion following DA stimulation, we designed an experiment to selectively eliminate surface-exposed $\mathrm{pH}-\mathrm{DRD} 2 \mathrm{~L}$ fluorescence before DA stimulation and TIRFM recording (Fig. 5A). We reason that if DRD2L originated from intracellular pools, photobleaching of preexisting surface $\mathrm{pH}-\mathrm{DRD} 2 \mathrm{~L}$ before DA stimulation would not affect the DA activity-dependent increase in DRD2L insertion (Fig. 5A, scenario a), because the intracellular pools of $\mathrm{pH}-$ DRD2L are nonfluorescent and hence are protected during photobleaching (Lin and Huganir, 2007). Conversely, if DRD2L originated from recycling of DRD2L that was initially present on the PM, photobleaching of surface $\mathrm{pH}-\mathrm{DRD} 2 \mathrm{~L}$ would abolish the 
DA activity-dependent increase in DRD2L insertion, as the recycled DRD2L would carry a photobleached pHluorin, and hence the insertion of $\mathrm{pH}-\mathrm{DRD} 2 \mathrm{~L}$ would be undetectable to our imaging system (Fig. $5 A$, scenario b). We first performed DA stimulation without photobleaching, which consistently enhanced $\mathrm{pH}-\mathrm{DRD} 2 \mathrm{~L}$ insertion frequency and amplitude (insertion frequency, $-\mathrm{DA}, 100 \pm 15 \%, n=14$ neurons; $+\mathrm{DA}$, $251 \pm 49 \%, n=15$ neurons; $p=0.0043$, Mann-Whitney test; insertion amplitude, $-\mathrm{DA}, 100 \pm 12 \%, n=68$ events from 14 neurons; $+\mathrm{DA}, 133 \pm 6 \%, n=222$ events from 15 neurons; $p<0.0001$, MannWhitney test) (Fig. 5B). However, photobleaching of preexisting surface $\mathrm{pH}$ DRD2L followed by $20 \mathrm{~min} 1 \mu \mathrm{M}$ DA stimulation resulted in significantly reduced DRD2L insertion frequency and amplitude (insertion frequency, $-\mathrm{DA}$, $100 \pm 19 \%, n=12$ neurons; $+\mathrm{DA}, 8 \pm$ $3 \%, n=12$ neurons; $p<0.0001$. MannWhitney test; insertion amplitude, -DA, $100 \pm 9 \%, n=86$ events from 12 neurons; + DA, $36 \pm 10 \%, n=7$ events from 12 neurons; $p=0.0046$, Mann-Whitney test) (Fig. $5 C$ ). This result suggests that DA-enhanced DRD2L insertion originated from recycling of endocytosed DRD2L. However, an alternative explanation for this reduced $\mathrm{pH}-\mathrm{DRD} 2 \mathrm{~L}$ insertion is that it resulted from damage to MSNs caused by photobleaching. To determine whether this alternative possibility could account for our observation, we performed the same photobleaching experiments, but recorded pH-DRD2L insertion immediately following the onset of DA stimulation instead of at the end of the $20 \mathrm{~min}$ stimulation period. Because immediately following photobleaching and the onset of DA stimulation no extensive mixing of photobleached surface $\mathrm{pH}$ DRD2L and existing intracellular pool of $\mathrm{pH}-\mathrm{DRD} 2 \mathrm{~L}$ has occurred, $\mathrm{pH}-\mathrm{DRD} 2 \mathrm{~L}$ insertion from preexisting intracellular pools in the constitutive recycling pathway should be detectable, if neurons are not damaged by our photobleaching protocol. Under this experimental condition, photobleaching of preexisting surface $\mathrm{pH}-\mathrm{DRD} 2 \mathrm{~L}$ did not reduce $\mathrm{pH}-\mathrm{DRD} 2 \mathrm{~L}$ insertion frequency ( $-\mathrm{DA}, 100 \pm 14 \%, n=12$ neurons; $+\mathrm{DA}, 97 \pm 28 \%$, $n=12$ neurons; $p=0.3526$, Mann-Whitney test), but slightly reduced the insertion amplitude ( $-\mathrm{DA}, 100 \pm 10 \%, n=69$ events from 12 neurons; +DA, $72 \pm 5 \%, n=67$ events from 12 neurons; $p=0.0477$, Mann-Whitney test), demonstrating that photobleaching does not damage MSNs (Fig. 5D). Together, our results strongly suggest that DA stimulation enhances DRD2L insertion via facilitating recycling of endocytosed DRD2L.

Recycling of DRD2L also depends on its endocytosis. Hypertonic sucrose, which blocks clathrin-dependent endocytosis, abolished the ability of DA stimulation to enhance DRD2L insertion frequency $(-\mathrm{DA}, 100 \pm 15 \%, n=46$ neurons; $+\mathrm{DA}, 183 \pm 20 \%, n=38$ neurons; $p=0.0002$ compared to $-\mathrm{DA} ;+\mathrm{DA}$ with sucrose, $38 \pm$ $7 \%, n=31$ neurons; $p<0.0001$ compared to + DA, Mann-Whitney test) but did not block the increase in insertion amplitude $(-\mathrm{DA}, 100 \pm 10 \%, n=249$ events from 46 neurons; $+\mathrm{DA}, 138 \pm$ $7 \%, n=419$ events from 38 neurons; $p<0.0001$ compared to $-\mathrm{DA}$; + DA with sucrose, $126 \pm 15 \%, n=70$ events from 31 neurons; $p=$ 0.0173 compared to $-\mathrm{DA}, p=0.2135$ compared to $+\mathrm{DA}$, MannWhitney test) (Fig. 6A). In addition, the pH-DRD2L $\mathrm{I}^{212} \mathrm{Y}^{213} \mathrm{I}^{214} \mathrm{~V}^{215}-\mathrm{A}^{212} \mathrm{~A}^{213} \mathrm{~A}^{214} \mathrm{~A}^{215}$ mutant that abolished arrestin binding and DRD2 endocytosis, but left DRD2 signaling through the G-protein pathways intact (Lan et al., 2009), also abolished the ability of DA stimulation to enhance DRD2L insertion (insertion frequency, $-\mathrm{DA}, 100 \pm 20 \%, n=28$ neurons; $+\mathrm{DA}, 211 \pm 28 \%, n=$ 19 neurons; $p=0.0008$ compared to $-\mathrm{DA} ;+\mathrm{DA}+\mathrm{IYIV} \_4 \mathrm{~A}, 16 \pm$ $6 \%, n=17$ neurons; $p<0.0001$ compared to $+\mathrm{DA}$; insertion amplitude, $-\mathrm{DA}, 100 \pm 12 \%, n=88$ events from 28 neurons; $+\mathrm{DA}$, $156 \pm 15 \%, n=162$ events from 20 neurons; $p<0.0015$ compared to $-\mathrm{DA} ;+\mathrm{DA}+\mathrm{DRD} 2$ IYIV_4A, $64 \pm 19 \%, n=8$ events from 17 
neurons; $p=0.1828$ compared to $-\mathrm{DA}, p=0.0286$ compared to +DA, Mann-Whitney test) (Fig. 6B). This result demonstrates that DA stimulation enhances DRD2L insertion via facilitating the endosomal recycling of DRD2L. Interestingly, Rab4DN did not affect the ability of DA stimulation to increase pH-DRD2L insertion (insertion frequency, $-\mathrm{DA}, 100 \pm 7 \%, n=65$ neurons; $+\mathrm{DA}, 188 \pm$ $15 \%, n=34$ neurons; $p<0.0001$ compared to $-\mathrm{DA}$; $+\mathrm{DA}$ + Rab4DN, $152 \pm 20 \%, n=23$ neurons; $p=0.1410$ compared to +DA; insertion amplitude, $-\mathrm{DA}, 100 \pm 4 \%, n=474$ events from 65 neurons; +DA, $153 \pm 6 \%, n=400$ events from 34 neurons; $p<$ 0.0001 compared to $-\mathrm{DA} ;+\mathrm{DA}+\mathrm{Rab} 4 \mathrm{DN}, 112 \pm 6 \%, n=236$ events from 23 neurons; $p=0.0002$ compared to $+\mathrm{DA}$, MannWhitney test) (Fig. 6C). In contrast, Rab11DN completely abolished the ability of DA stimulation to enhance $\mathrm{pH}-\mathrm{DRD} 2 \mathrm{~L}$ insertion (insertion frequency, +DA +Rab11DA, $90 \pm 11 \%, n=19$ neurons; $p<0.0001$ compared to $+\mathrm{DA}$; insertion amplitude, $+\mathrm{DA}$ + Rab11DN, $193 \pm 22 \%, n=88$ events from 19 neurons; $p=0.3737$ compared to $+\mathrm{DA}, p=0.0598$ compared to $\mathrm{pHDRD} 2 \mathrm{~L}$ + Rab11DN group in Fig. 2C, Mann-Whitney test) (Fig. 6C). Together, these results demonstrate that DA stimulation enhances DRD2L recycling primarily through Rab11-sensitive recycling endosomes.

In our imaging experiments, we observed two different modes of diffusion following DRD2L insertion: a relatively slow diffusion and a more rapid diffusion. In the presence of Rab4DN or Rab11DN, we did not observe selective elimination of either diffusion mode. However, we believe that the two different modes of diffusion represent diffusion dynamics of DRD2L only immediately following the insertion of these receptors to the $\mathrm{PM}$, and may not represent more general diffusion dynamics of DRD2L on the PM. We believe that techniques such as Q dot-based single-particle tracking will be more informative for determining surface dynamics of DRD2L than quantitatively comparing diffusion of DRD2L immediately following its insertion to the PM.

\section{Constitutive and activity-dependent DRD2L recycling differentially regulate surface levels of DRD2L}

We next examined surface expression levels of DRD2L to determine how disrupting endosomal recycling of DRD2L impacts its function. Under basal conditions, Rab4DN, but not Rab11DN, significantly reduced steady-state surface expression of DRD2L (control, $100 \pm 8 \%, n=79$ neurons; Rab4DN, $61 \pm 6 \%, n=64$ neurons; $p=0.0001$ compared to control; Rab11DN, $90 \pm 13 \%$, $n=45$ neurons; $p=0.1869$ compared to control, Mann-Whitney test) (Fig. 7A), suggesting that constitutive DRD2L recycling through the Rab4-sensitive fast recycling pathway is important in maintaining steady-state surface levels of DRD2L, while Rab11sensitive DRD2L recycling is dispensable under basal conditions. In contrast, when stimulated with DA, both the control group and the Rab4DN group displayed similar amounts of reduction in DRD2L surface levels (control, $100 \pm 8 \%, n=79$ neurons; DA, $50 \pm 5 \%, n=55$ neurons; $p<0.0001$ compared to control; Rab4DN/DA, $68 \pm 9 \%, n=36$ neurons; $p=0.1407$ compared to DA, Mann-Whitney test), while the Rab11DN group showed a significantly greater amount of reduction in DRD2L surface levels (Rab11DN/DA, $30 \pm 3 \%, n=42$ neurons; $p=0.0035$ compared to DA, Mann-Whitney test) (Fig. 7B). These results demonstrate that Rab11-sensitive DRD2L recycling is important for resensitization of DRD2L following DA stimulation. Together, these results underscore the distinct roles of constitutive and DA activity-dependent DRD2L recycling in regulation of DRD2L function (Fig. 7C).

\section{Discussion}

Technical advances in elucidating dynamic DRD2 insertion Dynamic trafficking of DRD2 between the PM and intracellular compartments is mediated by small vesicles, which contain only limited numbers of receptors. Therefore, elucidating the mechanisms governing dynamic trafficking of DRD2 requires experimental approaches with the sufficient spatial and temporal resolution necessary to detect single vesicles and to distinguish between receptors on the plasma membrane and those in intracellular compartments. Here we report an experimental approach that allows us to directly examine molecular mechanisms regulating DRD2 insertion to the PM in cultured MSNs. By tagging the $\mathrm{N}$ terminus of DRD2 with superecliptic pHluorin, and by imaging $\mathrm{pH}$-DRD2 using TIRFM, we were able to visualize individual vesicular insertion events of $\mathrm{pH}-\mathrm{DRD} 2$ in cultured MSNs. Insertion of pH-DRD2 in MSNs occurs on neuronal somatic and dendritic surfaces. Individual insertion events of $\mathrm{pH}$ DRD2 on average contains $30 \pm 1$ molecules. Lateral diffusion of DRD2 was observed following its insertion. $\mathrm{pH}$-DRD2S showed insertion frequencies and amplitudes in MSNs similar to those of pH-DRD2L, and pH-DRD2L showed similar properties of insertion in both mixed MSNs and $\mathrm{D}_{2}$-type MSNs. In addition, using our new approach, we showed that, under basal conditions, the observed pH-DRD2 insertion depended on DRD2 endocytosis, originated primarily from Rab4-sensitive sorting endosomes, and represented constitutive DRD2 recycling between sorting endosomes and the PM. We also showed that glutamatergic and GABAergic activities did not affect DRD2 insertion to the PM, and further identified a DA activity-dependent DRD2 recycling pathway through Rab11-sensitive recycling endosomes. Finally, we showed that these two endosomal recycling pathways of DRD2 are functionally distinct: the constitutive DRD2 recycling pathway determines steady-state surface levels of DRD2, and the activity-dependent DRD2 recycling pathway is important for functional resensitization of DRD2.

\section{The fate of DRD2 following endocytosis}

It is well known that upon DA stimulation, DRD2 desensitization occurs via DA-induced DRD2 endocytosis (Kim et al., 2001; Jeanneteau et al., 2004; Kabbani et al., 2004; Macey et al., 2004; Namkung and Sibley, 2004; Sugiura et al., 2004; Bartlett et al., 2005; Genedani et al., 2005; Torvinen et al., 2005; Paspalas et al., 2006; Iizuka et al., 2007; Kim, 2008; Xiao et al., 2009; Celver et al., 2010; Shimokawa et al., 2010). However, the fate of DRD2 following DA-induced endocytosis remains elusive. Using heterologous cells, DRD2 was shown to undergo agonist-independent and agonist-dependent endocytosis, and endocytosed DRD2 was shown to recycle back to the PM (Vickery and von Zastrow, 1999; Namkung et al., 2009). In contrast, Bartlett et al. (2005) showed that in heterologous cells DRD2 was primarily targeted to the degradation pathway following DA stimulation. Because both studies were performed using heterologous cells, it remains to be determined whether DRD2 undergoes both agonist-independent and agonist-dependent endocytosis in neurons, and what the fate of DRD2 is, in a neuron-specific cellular context, following DA-induced endocytosis.

Our current study using primary cultured MSNs clearly supports the existence of both agonist-independent and agonistdependent DRD2 endocytosis in MSNs, and provides direct evidence of the existence of both constitutive and DA activitydependent DRD2 recycling pathways. We further showed that these two DRD2 recycling pathways are differentially regulated: DRD2 internalized via agonist-independent endocytosis is recycled through the Rab4-sensitive fast recycling pathway, whereas 

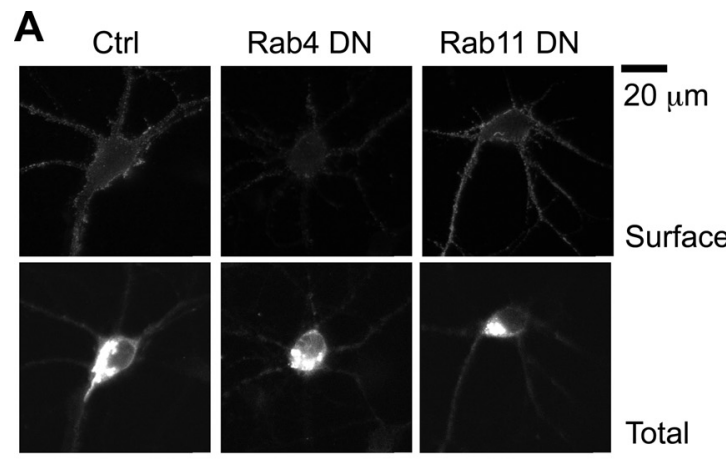

B Ctrl

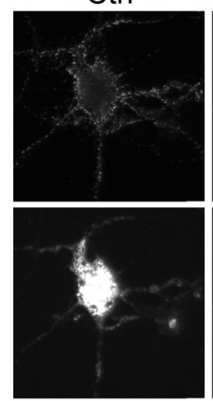

DA
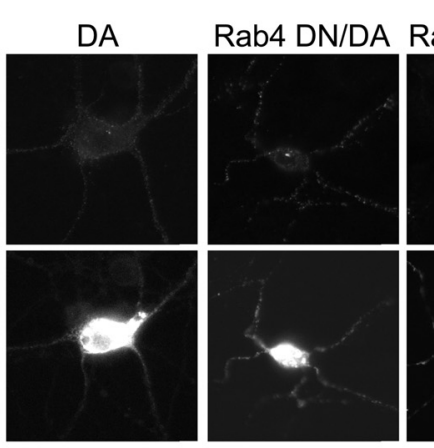

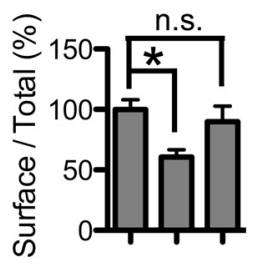

Rab4 DN - + -

Rab11 DN - - +

group $122 \quad 3$

C

\section{Basal conditions}

constitutive recycling

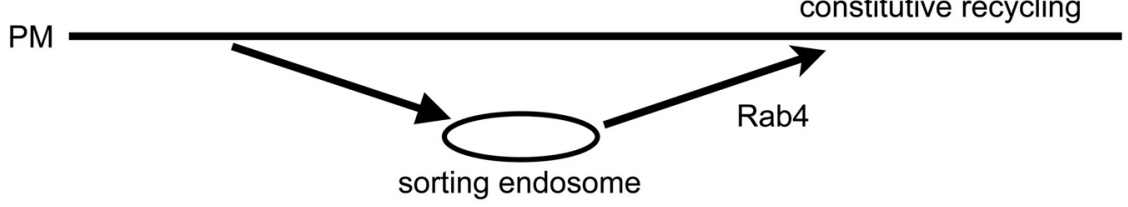

DA stimulation

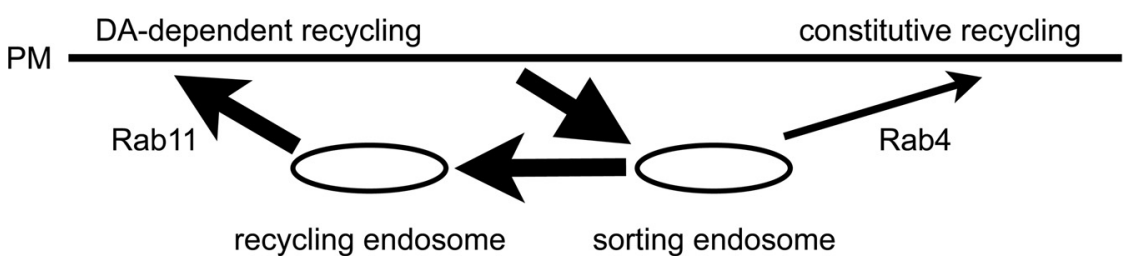

Figure 7. Rab4 and Rab11 differentially regulate DRD2L function. $\boldsymbol{A}$, Rab4DN reduces steady-state surface expression of DRD2L. Surface, Surface staining of pH-DRD2L; Total, total level of pH-DRD2L; group 1, control; group 2, Rab4DN; group 3, Rab11DN. B, Rab11DN inhibits DRD2L re-sensitization following DA stimulation. Group 1, Control; group 2, control plus DA; group 3, Rab4DN plus DA; group 4, Rab11DN plus DA. C, A model for the roles of Rab4 and Rab11 in regulation of DRD2L recycling. Under basal conditions, internalized DRD2L constitutively recycles from sorting endosomes to the PM via the Rab4-dependent route. Rab11 contributes little to constitutive DRD2L recycling. In contrast, when stimulated with DA, the Rab4-dependent fast recycling remains fully functional, but DRD2L recycling and functional resensitization occur primarily via the Rab11-dependent recycling pathway. Asterisks denote statistical significance compared to the control group. n.s., Not significant.

DRD2 internalized via agonist (DA)-dependent endocytosis is recycled primarily through the Rab11-sensitive slow recycling pathway. These two DRD2 recycling pathways play distinct roles in regulating DRD2 function in MSNs. We hypothesized that, under basal conditions, constitutive recycling of DRD2 through Rab4-dependent sorting endosomes serves as a quality control system: receptors are sorted toward either the PM via the Rab4-sensitive fast recycling pathway, or degradation pathways if specific modifications or damage to the receptor are detected. Disruption of this quality control system for DRD2 will either trap endocytosed DRD2 intracellularly, or target these receptors toward degradation pathways, consequently leading to decreased DRD2 function. In contrast, upon DA stimulation, Rab11-sensitive recycling endosomes play a prominent role in facilitating the functional resensitization of DRD2. Because
Rab11-sensitive endosomal recycling is a slow recycling pathway (Stenmark, 2009), DRD2 recycling through this slow recycling pathway for its resensitization may allow sufficient time for the desensitized receptors to undergo specific posttranslational modifications (Namkung et al., 2009) before they recycle back to the PM. Finally, given that the Rab11-dependent recycling endosomes supply glutamate receptors for the expression of synaptic plasticity in response to increased glutamatergic activity (Park et al., 2004), our findings further suggest that Rab11-dependent recycling endosomes may represent a general endosomal route for supplying increased number of specific receptors to the PM in response to an increase in specific neuronal activity.

Although we cannot provide a clear explanation for the difference in the fate of DRD2 following DA-induced endocytosis in 
heterologous cells observed by different groups, it is possible that different functional levels of Rab11-dependent recycling pathway under specific experimental conditions used by different groups may account for the discrepancy. Our findings provide strong evidence that Rab4 and Rab11 regulate endosomal recycling of DRD2. However, it is worth noting that direct evidence demonstrating DRD2 trafficking directly through Rab4 and Rab11 vesicles is still lacking. Further studies using other advanced optical imaging approaches such as dual-color live imaging with high spatial and temporal resolution or super-resolution microscopy will be important for detailed examination of DRD2 trafficking and sorting through different endosomal compartments.

\section{Insights gained from quantification of insertion amplitude}

Using our imaging approach, at least two parameters could be derived from our experiments: the frequency and the amplitude of insertion. The majority of our results reflect changes in insertion frequency, which in turn reflects the number of vesicles carrying $\mathrm{pH}-\mathrm{DRD} 2$ that are inserted to the PM. It is important to note that the insertion amplitude, which reflects the number of pH-DRD2 each vesicle carries, can also provide critical insights into how DRD2 trafficking is regulated. For example, upon DA stimulation, DRD2 insertion amplitude is significantly increased in addition to the increase in insertion frequency (Fig. $4 C$ ), and the degree of increase in amplitude is reduced when the Rab4dependent recycling pathway is blocked under DA stimulation (Fig. 6C). These results suggest that DA stimulation not only mobilizes vesicle insertion from Rab11-dependent recycling endosomes, but also facilitates loading of greater numbers of receptors into Rab4-dependent vesicles. However, this DA-stimulated loading of more DRD2 molecules into Rab4-dependent vesicles has a much smaller effect in facilitating DRD2 functional resensitization, as blocking the Rab4-dependent recycling pathway does not affect functional resensitization of DRD2 compared to the control group (Fig. 7B).

Our results by blocking Rab11-dependent recycling endosomes also suggest that the Rab4-dependent DRD2 recycling pathway remains fully functional under DA stimulation. This is also evident in our photobleaching experiments (Fig. 5). Following photobleaching of existing surface $\mathrm{pH}-\mathrm{DRD} 2$, and immediately following the onset of DA stimulation, the frequency of pH-DRD2 insertion remains unchanged but the amplitude of insertion is slightly reduced. This slight reduction in DRD2 insertion amplitude suggests that the constitutive recycling of DRD2 through the Rab4-dependent route is fast, such that by the time we perform our recording following photobleaching and DA stimulation, a small portion of DRD2 carrying photobleached pHluorin has entered the constitutive recycling route, leading to reduced number of fluorescent $\mathrm{pH}-\mathrm{DRD} 2$ in each vesicles.

Another example of gaining insight into regulation of DRD2 trafficking from insertion amplitude comes from the observation that the DRD2 insertion amplitude was significantly increased when the Rab11-dependent recycling pathway was blocked (Fig. $2 C)$. This observation suggests that under basal conditions, the number of available vesicle in the Rab4 route is limited; therefore, when the Rab11-dependent recycling pathway is blocked, increased numbers of DRD2 receptors are forced into each vesicle in the Rab4-dependent fast recycling route. Such insights from the quantification of insertion frequency and amplitude of DRD2 insertion cannot be obtained using existing biochemical methods, such as biotinylation or immunodetection of surfaceexposed receptor populations. Results from our current study and our previous studies using a similar optical imaging ap- proach to study the membrane insertion of glutamate receptor (Lin et al., 2009) clearly illustrate the power of this imaging strategy to dissect the detailed molecular and cellular mechanisms governing dynamic receptor trafficking and function. Our optical imaging approach offers excellent spatial and temporal resolution for studying the dynamic membrane insertion of receptors and will be a valuable addition to the collection of biochemical methods currently used to study membrane receptor trafficking and function.

In sum, our findings underscore the importance of endosomal recycling in the regulation of DRD2 function. Further dissecting the detailed molecular mechanisms governing constitutive and DA activity-dependent DRD2 recycling by integrating our optical imaging approach with other biochemical methods will shed light onto how DRD2 recycling through different endosomal compartments impacts its function in neurons, paving the way toward the goal of targeting DRD2 trafficking as a novel therapeutic strategy for psychiatric disorders with DRD2 dysfunction.

\section{References}

Bartlett SE, Enquist J, Hopf FW, Lee JH, Gladher F, Kharazia V, Waldhoer M, Mailliard WS, Armstrong R, Bonci A, Whistler JL (2005) Dopamine responsiveness is regulated by targeted sorting of D2 receptors. Proc Natl Acad Sci U S A 102:11521-11526.

Binda AV, Kabbani N, Lin R, Levenson R (2002) D2 and D3 dopamine receptor cell surface localization mediated by interaction with protein 4.1N. Mol Pharmacol 62:507-513.

Blum K, Sheridan PJ, Wood RC, Braverman ER, Chen TJ, Cull JG, Comings DE (1996) The D2 dopamine receptor gene as a determinant of reward deficiency syndrome. J R Soc Med 89:396-400.

Bunzow JR, Van Tol HH, Grandy DK, Albert P, Salon J, Christie M, Machida CA, Neve KA, Civelli O (1988) Cloning and expression of a rat D2 dopamine receptor cDNA. Nature 336:783-787.

Carlsson A (1988) The current status of the dopamine hypothesis of schizophrenia. Neuropsychopharmacology 1:179-186.

Celver J, Sharma M, Kovoor A (2010) RGS9-2 mediates specific inhibition of agonist-induced internalization of D2-dopamine receptors. J Neurochem 114:739-749.

Edelstein A, Amodaj N, Hoover K, Vale R, Stuurman N (2010) Computer control of microscopes using microManager. Current protocols in molecular biology, pp 14.20.1-14.20.17. Wiley Online Library.

Fetsko LA, Xu R, Wang Y (2003) Alterations in D1/D2 synergism may account for enhanced stereotypy and reduced climbing in mice lacking dopamine D2L receptor. Brain Res 967:191-200.

Free RB, Hazelwood LA, Cabrera DM, Spalding HN, Namkung Y, Rankin ML, Sibley DR (2007) D1 and D2 dopamine receptor expression is regulated by direct interaction with the chaperone protein calnexin. J Biol Chem 282:21285-21300.

Genedani S, Guidolin D, Leo G, Filaferro M, Torvinen M, Woods AS, Fuxe K, Ferré S, Agnati LF (2005) Computer-assisted image analysis of caveolin-1 involvement in the internalization process of adenosine A2Adopamine D2 receptor heterodimers. J Mol Neurosci 26:177-184.

Genedani S, Carone C, Guidolin D, Filaferro M, Marcellino D, Fuxe K, Agnati LF (2010) Differential sensitivity of A2A and especially D2 receptor trafficking to cocaine compared with lipid rafts in cotransfected $\mathrm{CHO}$ cell lines. Novel actions of cocaine independent of the DA transporter. J Mol Neurosci 41:347-357.

Holmes A, Lachowicz JE, Sibley DR (2004) Phenotypic analysis of dopamine receptor knockout mice; recent insights into the functional specificity of dopamine receptor subtypes. Neuropharmacology 47:1117-1134.

Iizuka Y, Sei Y, Weinberger DR, Straub RE (2007) Evidence that the BLOC-1 protein dysbindin modulates dopamine $\mathrm{D} 2$ receptor internalization and signaling but not D1 internalization. J Neurosci 27:12390-12395.

Jeanneteau F, Diaz J, Sokoloff P, Griffon N (2004) Interactions of GIPC with dopamine D2, D3 but not $\mathrm{D} 4$ receptors define a novel mode of regulation of G protein-coupled receptors. Mol Biol Cell 15:696-705.

Ji Y, Yang F, Papaleo F, Wang HX, Gao WJ, Weinberger DR, Lu B (2009) 
Role of dysbindin in dopamine receptor trafficking and cortical GABA function. Proc Natl Acad Sci U S A 106:19593-19598.

Kabbani N, Jeromin A, Levenson R (2004) Dynamin-2 associates with the dopamine receptor signalplex and regulates internalization of activated D2 receptors. Cell Signal 16:497-503.

Karam CS, Ballon JS, Bivens NM, Freyberg Z, Girgis RR, Lizardi-Ortiz JE, Markx S, Lieberman JA, Javitch JA (2010) Signaling pathways in schizophrenia: emerging targets and therapeutic strategies. Trends Pharmacol Sci 31:381-390.

Kenny PJ (2011) Reward mechanisms in obesity: new insights and future directions. Neuron 69:664-679.

Khan ZU, Mrzljak L, Gutierrez A, de la Calle A, Goldman-Rakic PS (1998) Prominence of the dopamine D2 short isoform in dopaminergic pathways. Proc Natl Acad Sci U S A 95:7731-7736.

Kim JH, Cho EY, Min C, Park JH, Kim KM (2008a) Characterization of functional roles of DRY motif in the 2nd intracellular loop of dopamine D2 and D3 receptors. Arch Pharm Res 31:474-481.

Kim KM, Valenzano KJ, Robinson SR, Yao WD, Barak LS, Caron MG (2001) Differential regulation of the dopamine D2 and D3 receptors by G protein-coupled receptor kinases and beta-arrestins. J Biol Chem 276:37409-37414.

Kim OJ (2008) A single mutation at lysine 241 alters expression and trafficking of the D2 dopamine receptor. J Recept Signal Transduct Res 28:453-464.

Kim OJ, Ariano MA, Namkung Y, Marinec P, Kim E, Han J, Sibley DR (2008b) D2 dopamine receptor expression and trafficking is regulated through direct interactions with ZIP. J Neurochem 106:83-95.

Lan H, Liu Y, Bell MI, Gurevich VV, Neve KA (2009) A dopamine D2 receptor mutant capable of $\mathrm{G}$ protein-mediated signaling but deficient in arrestin binding. Mol Pharmacol 75:113-123.

Lawford BR, Young R, Noble EP, Kann B, Ritchie T (2006) The D2 dopamine receptor (DRD2) gene is associated with co-morbid depression, anxiety and social dysfunction in untreated veterans with post-traumatic stress disorder. Eur Psychiatry 21:180-185.

Lin DT, Huganir RL (2007) PICK1 and phosphorylation of the glutamate receptor 2 (GluR2) AMPA receptor subunit regulates GluR2 recycling after NMDA receptor-induced internalization. J Neurosci 27:13903-13908.

Lin DT, Makino Y, Sharma K, Hayashi T, Neve R, Takamiya K, Huganir RL (2009) Regulation of AMPA receptor extrasynaptic insertion by $4.1 \mathrm{~N}$, phosphorylation and palmitoylation. Nat Neurosci 12:879-887.

Lin R, Karpa K, Kabbani N, Goldman-Rakic P, Levenson R (2001) Dopamine D2 and D3 receptors are linked to the actin cytoskeleton via interaction with filamin A. Proc Natl Acad Sci U S A 98:5258-5263.

Lin R, Canfield V, Levenson R (2002) Dominant negative mutants of filamin A block cell surface expression of the D2 dopamine receptor. Pharmacology 66:173-181.

Lindgren N, Usiello A, Goiny M, Haycock J, Erbs E, Greengard P, Hokfelt T, Borrelli E, Fisone G (2003) Distinct roles of dopamine D2L and D2S receptor isoforms in the regulation of protein phosphorylation at presynaptic and postsynaptic sites. Proc Natl Acad Sci U S A 100:4305-4309.

Luscher B, Fuchs T, Kilpatrick CL (2011) GABAA receptor traffickingmediated plasticity of inhibitory synapses. Neuron 70:385-409.

Macey TA, Gurevich VV, Neve KA (2004) Preferential Interaction between the dopamine D2 receptor and Arrestin2 in neostriatal neurons. Mol Pharmacol 66:1635-1642.

Mack KJ, O’Malley KL, Todd RD (1991) Differential expression of dopaminergic D2 receptor messenger RNAs during development. Brain Res Dev Brain Res 59:249-251.

Marley A, von Zastrow M (2010) Dysbindin promotes the post-endocytic sorting of G protein-coupled receptors to lysosomes. PLoS One 5:e9325.

Matsuda T, Cepko CL (2007) Controlled expression of transgenes introduced by in vivo electroporation. Proc Natl Acad Sci U S A 104:1027-1032.

Miesenböck G, De Angelis DA, Rothman JE (1998) Visualizing secretion and synaptic transmission with $\mathrm{pH}$-sensitive green fluorescent proteins. Nature 394:192-195.

Missale C, Nash SR, Robinson SW, Jaber M, Caron MG (1998) Dopamine receptors: from structure to function. Physiol Rev 78:189-225.
Namkung Y, Sibley DR (2004) Protein kinase C mediates phosphorylation, desensitization, and trafficking of the D2 dopamine receptor. J Biol Chem 279:49533-49541.

Namkung Y, Dipace C, Javitch JA, Sibley DR (2009) G protein-coupled receptor kinase-mediated phosphorylation regulates post-endocytic trafficking of the D2 dopamine receptor. J Biol Chem 284:15038-15051.

Neve KA, Neve RL, Fidel S, Janowsky A, Higgins GA (1991) Increased abundance of alternatively spliced forms of D2 dopamine receptor mRNA after denervation. Proc Natl Acad Sci U S A 88:2802-2806.

Park M, Penick EC, Edwards JG, Kauer JA, Ehlers MD (2004) Recycling endosomes supply AMPA receptors for LTP. Science 305:1972-1975.

Park SK, Nguyen MD, Fischer A, Luke MP, Affar el B, Dieffenbach PB, Tseng HC, Shi Y, Tsai LH (2005) Par-4 links dopamine signaling and depression. Cell 122:275-287.

Paspalas CD, Rakic P, Goldman-Rakic PS (2006) Internalization of D2 dopamine receptors is clathrin-dependent and select to dendro-axonic appositions in primate prefrontal cortex. Eur J Neurosci 24:1395-1403.

Rouge-Pont F, Usiello A, Benoit-Marand M, Gonon F, Piazza PV, Borrelli E (2002) Changes in extracellular dopamine induced by morphine and cocaine: crucial control by D2 receptors. J Neurosci 22:3293-3301.

Shepherd JD, Huganir RL (2007) The cell biology of synaptic plasticity: AMPA receptor trafficking. Annu Rev Cell Dev Biol 23:613-643.

Shimokawa N, Haglund K, Hölter SM, Grabbe C, Kirkin V, Koibuchi N, Schultz C, Rozman J, Hoeller D, Qiu CH, Londoño MB, Ikezawa J, Jedlicka P, Stein B, Schwarzacher SW, Wolfer DP, Ehrhardt N, Heuchel R, Nezis I, Brech A, et al. (2010) CIN85 regulates dopamine receptor endocytosis and governs behaviour in mice. EMBO J 29:2421-2432.

Stenmark H (2009) Rab GTPases as coordinators of vesicle traffic. Nat Rev Mol Cell Biol 10:513-525.

Sugiura H, Iwata K, Matsuoka M, Hayashi H, Takemiya T, Yasuda S, Ichikawa M, Yamauchi T, Mehlen P, Haga T, Yamagata K (2004) Inhibitory role of endophilin 3 in receptor-mediated endocytosis. J Biol Chem 279:23343-23348.

Sun X, Milovanovic M, Zhao Y, Wolf ME (2008) Acute and chronic dopamine receptor stimulation modulates AMPA receptor trafficking in nucleus accumbens neurons cocultured with prefrontal cortex neurons. J Neurosci 28:4216-4230.

Tirotta E, Fontaine V, Picetti R, Lombardi M, Samad TA, Oulad-Abdelghani M, Edwards R, Borrelli E (2008) Signaling by dopamine regulates D2 receptors trafficking at the membrane. Cell Cycle 7:2241-2248.

Torvinen M, Torri C, Tombesi A, Marcellino D, Watson S, Lluis C, Franco R, Fuxe K, Agnati LF (2005) Trafficking of adenosine A2A and dopamine D2 receptors. J Mol Neurosci 25:191-200.

Usiello A, Baik JH, Rougé-Pont F, Picetti R, Dierich A, LeMeur M, Piazza PV, Borrelli E (2000) Distinct functions of the two isoforms of dopamine D2 receptors. Nature 408:199-203.

Vickery RG, von Zastrow M (1999) Distinct dynamin-dependent and -independent mechanisms target structurally homologous dopamine receptors to different endocytic membranes. J Cell Biol 144:31-43.

Volkow ND, Fowler JS, Wang GJ, Baler R, Telang F (2009) Imaging dopamine's role in drug abuse and addiction. Neuropharmacology 56 [Suppl 1]:3-8.

Wang GJ, Volkow ND, Fowler JS (2002) The role of dopamine in motivation for food in humans: implications for obesity. Expert Opin Ther Targets 6:601-609.

Wise RA (2008) Dopamine and reward: the anhedonia hypothesis 30 years on. Neurotoxicity research 14:169-183.

Xiao MF, Xu JC, Tereshchenko Y, Novak D, Schachner M, Kleene R (2009) Neural cell adhesion molecule modulates dopaminergic signaling and behavior by regulating dopamine $\mathrm{D}_{2}$ receptor internalization. J Neurosci 29:14752-14763.

Xu R, Hranilovic D, Fetsko LA, Bucan M, Wang Y (2002) Dopamine D2S and D2L receptors may differentially contribute to the actions of antipsychotic and psychotic agents in mice. Mol Psychiatry 7:1075-1082.

Yudowski GA, Puthenveedu MA, von Zastrow M (2006) Distinct modes of regulated receptor insertion to the somatodendritic plasma membrane. Nat Neurosci 9:622-627. 